American Journal of Environmental Sciences 6 (2): 191-203, 2010

ISSN 1553-345X

(C) 2010 Science Publications

\title{
Temperature Trends and Distribution in the Arabian Peninsula
}

\author{
${ }^{1}$ Mohammad N. ElNesr, ${ }^{2}$ Majed M. Abu-Zreig and ${ }^{1}$ Abdurrahman A. Alazba \\ ${ }^{1}$ Alamoudi Water Chair, King Saud University, P.O. Box 2460, Riyadh 11451, Saudi Arabia \\ ${ }^{2}$ Department of Civil Engineering, Jordan University of Science and Technology, \\ Irbid, Jordan
}

\begin{abstract}
Problem statement: Temperature trend's investigation is important for proper water resources management and urban planning. This study aims to investigate trends and distribution of temperature in the past thirty years for the Kingdom of Saudi Arabia (KSA), which represents about 86\% of the Arabian Peninsula's area. Approach: The trend in the temperature time series, including the recorded maximum, average and minimum daily values, were analyzed using non-parametric statistics. These were Mann-Kendall tau coefficient and Sen's slope estimator. Results: (1): The study showed a warming trend through 9 months of the year except in November to January where nonsignificant cooling trends were observed. (2): The most significant warming trend appears in the summer months of June, August and September around the central region of KSA. (3) Spatially, The northwestern and southern regions were the least affected by the warming trend. Conclusion: The study concluded that KSA as well as the Arabian Peninsula are suffering from a considerable warming temperature trend, which is an important issue to be considered for rural development and water resources management.
\end{abstract}

Key words: Temperature trend, time series, spatial distribution, Arabian Peninsula, Saudi Arabia

\section{INTRODUCTION}

The earth's climate is unstable; urbanization and greenhouse effect climate change in non-predictable manner (Can and Atimtay, 2004). Climate change was defined by Landsberg (1975) as a shift in climatic conditions to a new equilibrium position with significantly changing values of the climatic-elements. Climate change highly influences human life in municipal and agricultural fields. Most of the climate factors, like temperature and relative humidity, affect plant water demand. However, investigating changes in trend and magnitude of each climate factor will help in planning and management of agricultural systems. For that, the climate change phenomenon was studied extensively in the last decades in both regional and global scopes (Schwartz and Randall, 2003).

Climate change research has been conducted in many parts of the world. Cueto et al. (2009) performed a spatial and temporal analysis of air temperature in Mexicali, B. C. in Mexican Republic. Their study differentiates between urban and rural areas; they found that the increasing trend in temperature is more in urban areas than rural areas, thus highlighting the effect of urbanization as a source for global warming.
Conversely, Xu et al. (2006) found a significant decreasing trend in both the reference evapotranspiration and the pan evaporation through a forty-year study of changing region in China. They referred this decrease to the significant reduction in the net total radiation over the region, which might be caused by the heavy cloudiness near Yangtze River. They also performed a sensitivity analysis that showed that the reference evapotranspiration is most sensitive to the net total radiation, followed by relative humidity, air temperature and wind speed. Not far from that, in India, Govinda (1993) had evaluated the long-term changes of seasonal and annual surface air temperatures and precipitation over Mahanadi river basin. He reported that there is a highly significant increasing trend in temperature values, while rainfall did not show any significant trend in the same period.

Due to its hot climate and water scarcity problems, the Arabian Peninsula (AP) is sensitive to climate variability, especially those affecting water demand. The Kingdom of Saudi Arabia (KSA) occupies about $86 \%$ of the area of the Arabian Peninsula (Arabia, 2009). In addition, the KSA is spread in all the directions of the AP except the southern region; hence, KSA climate could be considered a suitable

Corresponding Author: Mohammad N. ElNesr, Alamoudi Water Chair, King Saud University, P.O. Box 2460, Riyadh 11451, Saudi Arabia Tel: 966544909445 
representative for AP climate (Abderrahman and AlHarazin, 2008). Several studies have reported that the Middle East region may face more aridity due to temperature increase and rainfall decrease. Nasrallah and Balling (1995) found a statistically significant temperature increase of $0.07^{\circ} \mathrm{C} /$ decade over Kuwait during the period of 1950-1990. In Jordan, located on the northern part of the Arabian Peninsula, Smadi (2006) performed a study to trace changes in annual and seasonal temperature during the 20th century. His results showed a warming trend starting from the years 1957 and 1967 for the minimum and maximum temperatures respectively. In another study, Smadi and Zghoul (2006) reported that the total rainfall showed a decreasing trend starting from the year 1957 and forward. This means that as water demand increases due to continuous temperature rise, rainfall (and therefore water supply) decreases leading to complex water crisis. Similarly, Bou-Zeid and El-Fadel (2002) conducted a wide study covering the whole Middle East region. They found that water balance would be highly affected by the increasing temperature trend at a rate of $0.6-2.1^{\circ} \mathrm{C}$. Recently, Al-Zawad (2008) studied the impacts of climate change on water resources in KSA using the climate model "PRECIS". The results of this model were fairly correlated to the historic climatic data of the region. He concluded that the temperature, the evaporation and the wind speed have showed an increasing trend on all regions of the country.

However, previous studies were limited in scope and use the annual average data rather that monthly data. In addition, the applications of non-parametric statistics to detect trends in temperature have not been investigated deeply for the Saudi Arabia. Therefore, the aim of this study is to examine changes in temperature trend and magnitude in Saudi Arabia as a suitable representative of the Arabian Peninsula. The analyses of temperature include the recorded maximum, average and minimum daily value. The non-parametric statistics used in this study were Mann-Kendall coefficient and some Sen's slope indices.

\section{MATERIALS AND METHODS}

KSA geography and climatic database: Saudi Arabia lies between latitudes $16^{\circ} 21^{\prime} 58^{\prime \prime} \mathrm{N}$ and $32^{\circ} 9^{\prime} 57^{\prime \prime} \mathrm{N}$ and longitudes $34^{\circ} 33^{\prime} 48^{\prime \prime} \mathrm{E}$ and $55^{\circ} 41^{\prime} 29^{\prime \prime} \mathrm{E}$, as illustrated in Fig. 1. According to the Food and Agriculture Organization Statistics, the country falls in the tropical and subtropical desert region with dry climate and high temperatures in most of the region. Winds reaching the country are generally dry and almost all the area is arid. Because of the aridity and the relatively cloudless skies, there are large variations in daily temperature as well as between seasons and regions.

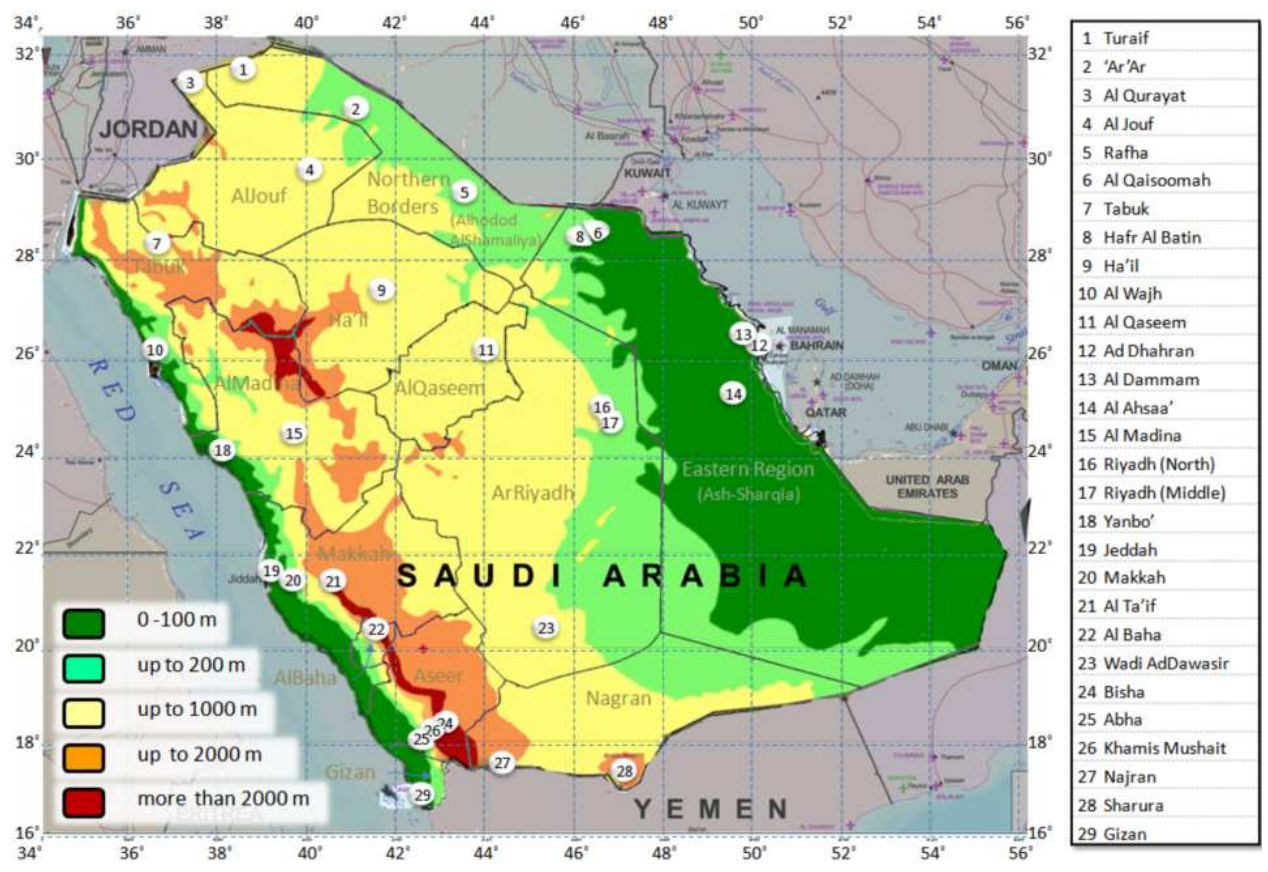

Fig. 1: Geographic and Topographic map of Saudi Arabia, showing 13 districts and 29 meteorological stations. Sources: Base map: National Imagery and Mapping Agency of the US Government and Districts: Ministry of Municipal and Rural Affairs. Topography: Albakry et al. (2010) 
Am. J. Environ. Sci., 6 (2): 191-203, 2010

Table 1: Geographical information of the meteorological stations included in this study

\begin{tabular}{|c|c|c|c|c|c|c|c|}
\hline \multirow[b]{2}{*}{ District } & \multicolumn{2}{|c|}{ Station } & \multicolumn{3}{|c|}{ Station coordinates } & \multirow[b]{2}{*}{ Logged years } & \multirow{2}{*}{$\begin{array}{l}\text { Average } \\
\text { temperature }{ }^{\circ} \mathrm{C}\end{array}$} \\
\hline & ID & Name & Latitude deg. N & Longitude deg. East & Altitude m & & \\
\hline Northern & 1 & Turaif & 31.41 & 38.40 & 818 & 29 & $19.06 \pm 8.27$ \\
\hline \multirow[t]{2}{*}{ Borders } & 2 & Arar & 31.00 & 41.00 & 600 & 29 & $22.01 \pm 9.17$ \\
\hline & 5 & Rafha & 29.38 & 43.29 & 447 & 29 & $23.33 \pm 9.05$ \\
\hline \multirow[t]{2}{*}{ AlJouf } & 3 & Guraiat & 31.50 & 37.50 & 560 & 4 & $20.04 \pm 7.91$ \\
\hline & 4 & Al Jouf & 29.47 & 40.06 & 671 & 29 & $22.03 \pm 8.61$ \\
\hline \multirow[t]{2}{*}{ Tabuk } & 7 & Tabuk & 28.22 & 36.38 & 776 & 29 & $21.99 \pm 7.53$ \\
\hline & 10 & Wejh & 26.12 & 36.28 & 21 & 29 & $25.00 \pm 3.98$ \\
\hline Ha'il & 9 & Hail & 27.26 & 41.41 & 1013 & 29 & $22.47 \pm 8.20$ \\
\hline AlQaseem & 11 & Gassim & 26.18 & 43.46 & 650 & 29 & $24.94 \pm 8.26$ \\
\hline Eastern & 6 & Qaisumah & 28.32 & 46.13 & 358 & 29 & $25.23 \pm 9.28$ \\
\hline \multirow[t]{4}{*}{ Region } & 8 & Hafr Al-Batin & 28.20 & 46.07 & 360 & 19 & $25.26 \pm 9.21$ \\
\hline & 12 & Dhahran & 26.16 & 50.10 & 17 & 29 & $26.48 \pm 7.49$ \\
\hline & 13 & Dammam & 26.42 & 50.12 & 1 & 9 & $26.72 \pm 7.86$ \\
\hline & 14 & Ahsa & 25.30 & 49.48 & 179 & 24 & $27.26 \pm 8.27$ \\
\hline \multirow[t]{3}{*}{ Riyadh } & 16 & Riyadh North & 24.42 & 46.44 & 611 & 24 & $25.80 \pm 8.12$ \\
\hline & 17 & Riyadh Middle & 24.63 & 46.77 & 624 & 29 & $26.66 \pm 8.10$ \\
\hline & 23 & W-Dawasir & 20.50 & 45.16 & 652 & 24 & $28.15 \pm 7.01$ \\
\hline \multirow{2}{*}{ Madina } & 15 & Madina & 24.33 & 39.42 & 636 & 29 & $28.45 \pm 7.00$ \\
\hline & 18 & Yenbo & 24.09 & 38.04 & 6 & 29 & $27.56 \pm 4.72$ \\
\hline \multirow{3}{*}{ Makkah } & 19 & Jeddah & 21.30 & 39.12 & 17 & 29 & $28.23 \pm 3.50$ \\
\hline & 20 & Makkah & 21.40 & 39.85 & 213 & 24 & $30.78 \pm 4.57$ \\
\hline & 21 & Taif & 21.29 & 40.33 & 1454 & 29 & $22.92 \pm 5.12$ \\
\hline Baha & 22 & Baha & 20.30 & 41.63 & 1652 & 24 & $22.83 \pm 4.92$ \\
\hline \multirow[t]{3}{*}{ Aseer } & 24 & Bisha & 19.59 & 42.37 & 1163 & 29 & $25.69 \pm 5.40$ \\
\hline & 25 & Abha & 18.14 & 42.39 & 2093 & 29 & $18.60 \pm 3.72$ \\
\hline & 26 & Khamis Mushait & 8.18 & 42.48 & 2057 & 29 & $9.49 \pm 3.75$ \\
\hline \multirow[t]{2}{*}{ Nagran } & 27 & Nejran & 17.37 & 44.26 & 1210 & 29 & $25.51 \pm 5.54$ \\
\hline & 28 & Sharurrah & 17.47 & 47.11 & 725 & 24 & $28.57 \pm 5.81$ \\
\hline Gizan & 29 & Gizan & 16.54 & 42.35 & 3 & 29 & $30.22 \pm 2.80$ \\
\hline
\end{tabular}

Climate data was taken from the Presidency of Meteorology and Environment (PME) in KSA. Data represents 29 meteorological stations covering all the 13 districts of the KSA, as shown in Fig. 1. The data represents a 29 years of daily meteorological parameters, from 1980-2008, for 20 stations, 24 years of data for 6 stations and 3 stations with less than 20 years as summarized in Table 1. The raw data was reported on daily basis from which the average monthly and yearly values were calculated. The data set is the most reliable one in KSA and used by all other governmental and academic agencies for climate research and prediction. Weather stations are equipped with up-to-date monitoring devices and subjected to regular inspection and replacement for defected devices (personal communication with the PME).

Statistical measures: Time-series analysis was adopted in this research to detect any trends in the meteorological parameters under study. The nonparametric statistics known as Man-Kendall (Kendall, 1975; Gilbert, 1987) and Sen slope (Sen, 1968) will be used in this research as stated below:

Kendall statistics: The Mann-Kendall test is a nonparametric test used for identifying trends in a time series data. The test compares the relative magnitudes of sample data rather than the data values themselves Both Kendall tau coefficient $(\tau)$ and Mann-Kendall score (s) are nonparametric statistics used to find rank correlation. Kendall $\tau$ is a ratio between the actual rating score of correlation, to the maximum possible score. To obtain the rating score for a time series, the dataset is sorted in ascending order according to time and then the following formula is applied:

$S=\sum_{j=1}^{j=n-1} \sum_{i=j+1}^{i=n} \operatorname{Sign}\left(x_{i}-x_{j}\right)$

Where:

$\mathrm{s} \quad=$ The rating score (also called the MannKendall sum)

$\mathrm{x}=$ The data value

$\mathrm{i}$ and $\mathrm{j}=$ Counters

$\mathrm{n} \quad=$ Number of data values

Sign $=\mathrm{A}$ function having values of $+1,0$, or -1 if $\left(\mathrm{x}_{\mathrm{i}}-\mathrm{x}_{\mathrm{j}}\right)$ is positive zero, or negative, respectively

According to this formula, the maximum value of $\mathrm{s}$ is:

$\mathrm{S}_{\max }=\frac{1}{2} \mathrm{n}(\mathrm{n}-1)$ 
Hence, the Kendall tau is calculated as:

$$
\tau=\frac{\mathrm{S}}{\mathrm{S}_{\max }}
$$

A positive value of $\mathrm{s}$ or $\tau$ is an indicator of an increasing trend and a negative value indicates a decreasing trend. However, it is necessary to compute the probability associated with $\mathrm{s}$ or $\tau$ and the sample size, $n$, to statistically quantify the significance of the trend. Kendall and Gibbons (1990) introduced a normal-approximation test that could be applied on datasets with more than ten values. After calculating s, the variance $\left(\sigma^{2}\right)$ of $s$ is calculated:

$$
\sigma^{2}=\frac{1}{18} n(n-1)(2 n+5)-C F_{R}
$$

$$
\mathrm{CF}_{\mathrm{R}}=\frac{1}{18} \sum_{\mathrm{k}=1}^{\mathrm{g}} \mathrm{m}_{\mathrm{k}}\left(\mathrm{m}_{\mathrm{k}}-1\right)\left(2 \mathrm{~m}_{\mathrm{k}}+5\right)
$$

Where:

$\mathrm{CF}_{\mathrm{R}}=$ Repetition correction factor, to fix the effect of tied groups of data (when some of the data values appear more than one time in the dataset, this group of values are called a tied group)

$\mathrm{g}=$ Number of tied groups

$\mathrm{k}=\mathrm{A}$ counter

$\mathrm{m}=$ Count of data values in each tied group

Next, the normal distribution parameter (called the Mann-Kendall statistic, Z) is calculated as follows:

$$
\mathrm{Z}= \begin{cases}\frac{1}{\sigma}(\mathrm{s}-1) & \rightarrow \mathrm{s}>0 \\ 0 & \rightarrow \mathrm{s}=0 \\ \frac{1}{\sigma}(\mathrm{s}+1) & \rightarrow \mathrm{s}<0\end{cases}
$$

The last step is to find the minimum level at which the parameter $\mathrm{Z}$ is significant as mentioned by Abramowitz and Stegun (1972):

$$
\alpha_{\min }=\left(\mathrm{b}_{0} \mathrm{e}^{-0.5 \mathrm{Z}^{2}}\right) \sum_{\mathrm{q}=1}^{\mathrm{q}=5} \mathrm{~b}_{\mathrm{q}} \cdot\left(1+\mathrm{b}_{6} \mathrm{ABS}(\mathrm{Z})\right)^{-\mathrm{q}}
$$

Where:

$$
\begin{aligned}
\alpha_{\min }= & \text { Minimum level of significance } \\
\mathrm{q}= & \text { Counter } \\
\mathrm{b}_{\mathrm{q}}= & \text { Constants: } \mathrm{b}_{0}=0.3989, \quad \mathrm{~b}_{1}=0.3194, \\
& \mathrm{~b}_{2}=-0.3566, \mathrm{~b}_{3}=1.7814, \mathrm{~b}_{4}=-1.8213, \\
& \mathrm{~b}_{5}=1.3303, \mathrm{~b}_{6}=0.2316
\end{aligned}
$$

$\mathrm{ABS}(\mathrm{Z})=$ The absolute value of $\mathrm{Z}$
Sen's slope estimator: Sen's statistic is the median slope of each point-pair slope in a dataset (Sen, 1968). To perform the complete Sen's test, the following rules and conditions are applied:

- The time series should be equally spaced, i.e., the interval between data points should be equal. However, Sen's method considers missing data

- Data should be sorted in ascending order according to time, then apply the following formula to calculate Sen's slope estimator $(\mathrm{Q})$ as the median of Sen's matrix members:

$$
Q=\operatorname{Median}\left\{\left[\left[\frac{x_{i}-x_{j}}{i-j}\right]_{j=1}^{j=n-1}\right]_{i=j+1}^{i=n}\right\}
$$

$\mathrm{Q}$ is the main component of Sen's statistics. Its sign reflects data trend direction, while its value indicates the steepness of the trend

- To determine whether the median slope is statistically different than zero, one should obtain the confidence interval of $\mathrm{Q}$ at specific probability level, e.g., 95\%. Assuming that $Q$ is normally distributed then the value of $\mathrm{Z}$ under two-tailed normal distribution curve is calculated at the level (1- $\alpha / 2)$, where $\mathrm{a}=1$-confedence level, e.g., $\mathrm{Z}$ $=1.96$. The variance of $\mathrm{Q}$ is calculated using Eq. 4 and a statistic $\mathrm{C}_{\mathrm{a}}$ is now calculated as follows:

$$
\mathrm{C}_{\mathrm{a}}=\mathrm{Z}_{1-\mathrm{a} / 2} \sqrt{\sigma^{2}}
$$

- The upper and lower boundary limits of the slope $\mathrm{Q}$ are calculated as follows:

$$
\begin{aligned}
& \mathrm{M}_{\mathrm{u}}=\operatorname{int}\left(0.5\left(\mathrm{n}_{\mathrm{q}}-\mathrm{C}_{\mathrm{a}}\right)\right) \\
& \mathrm{M}_{1}=\operatorname{int}\left(0.5\left(\mathrm{n}_{\mathrm{q}}+\mathrm{C}_{\mathrm{a}}\right)\right)+1
\end{aligned}
$$

Where:

$$
\begin{aligned}
\mathrm{M}_{\mathrm{u}} \text { and } \mathrm{M}_{\mathrm{l}}= & \text { The upper and lower boundaries. } \\
& \text { positions, respectively } \\
\mathrm{n}_{\mathrm{q}} & =\begin{array}{l}
\text { The number of members of Sen's matrix } \\
\text { that was produced by Eq. } 8 ; \mathrm{n}_{\mathrm{q}}=\mathrm{n}(\mathrm{n}-1)
\end{array}
\end{aligned}
$$

The slope Q is statistically different than zero if the two limits have similar sign.

Data analysis: Climatic data obtained for 29 stations for the past 29 years were used to calculate manKendall coefficients and Sen's slope estimator for maximum, average and minimum temperature for each 
month. Significance tests were also performed for $\tau$ and Q for each combination of station (29 station), month (12 months) and temperature parameters (3 parameters). Due to Sen and Kendall methods regulations, two stations were excluded from the analysis as their number of recorded years were less than 10 years. These stations are "3-Gurayat".and " 13 Dammam". The rest of the stations were analyzed as mentioned. Contour plots were used to view and compare trends in several months. Drawing a contour plot requires three factors, namely, longitude, latitude and a selected statistical parameter. Twelve contourplots, one for each month, were constructed for the maximum temperature. The data was gridded separately using the ordinary-point Kriging method (Isaaks and Srivastava, 1990). The resulted grid was blanked outside the political borders of the KSA. The political borders information of the KSA was grabbed from electronic map from the Saudi Ministry of Municipal and Rural Affairs The electronic map was digitized and converted to DMS geographic coordinate system. The blanked grid was plotted as a contour map using Surfer ${ }^{\mathrm{TM}} 8.0$ software (Surfer, 2002).

\section{RESULTS}

The daily temperature data for the 29 weather stations were analyzed for the past 30 years and the mean monthly and annual temperatures were calculated for the minimum, average and maximum daily temperatures. The mean monthly values of the average temperature varied from about $15 \mathrm{C}$ in January and rise to as high as 33 in July, which is the hottest month in the year, then temperatures decline again to reach $16 \mathrm{C}$ in December, as shown in Fig. 2. The mean maximum and minimum temperatures in July varied from 25 to as high as 40, respectively. The corresponding temperatures in the coolest month of the year, January, were 9 and $21^{\circ} \mathrm{C}$, respectively. Figure 2 shows the high variability in temperatures during the year and as well as during the month that make regional planning for water resources and agricultural production even $\mathrm{m}$ ore challenging.

Further analysis was carried out to examine the relationships between mean annual temperatures and stations altitudes and latitudes and the results are shown in Fig. 3 and 4, respectively. As expected from Fig. 3 temperature is inversely proportional to altitudes. Temperatures varied from about $30^{\circ} \mathrm{C}$ in sea level stations like Yenbo to as low as $18^{\circ} \mathrm{C}$ in Abha with altitude of about $2000 \mathrm{~m}$ above sea level. A linear relationship can approximate the relationship between $\mathrm{Ta}$ and altitude with a slope of $-0.004^{\circ} \mathrm{C} \mathrm{m}^{-1}$ and moderate $\mathrm{R}^{2}=0.4$. The standard deviations of Ta, shown in Fig. 3, were relatively constant at about $6^{\circ} \mathrm{C}$, even though temperature variations seemed to be relatively lower in high altitude compared to low altitude stations.

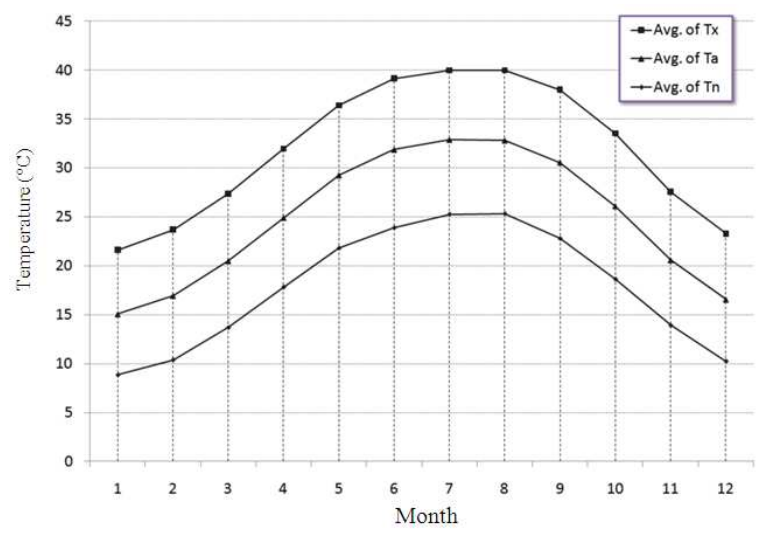

Fig. 2: The mean-monthly maximum, average and minimum temperature variations during the year in SA

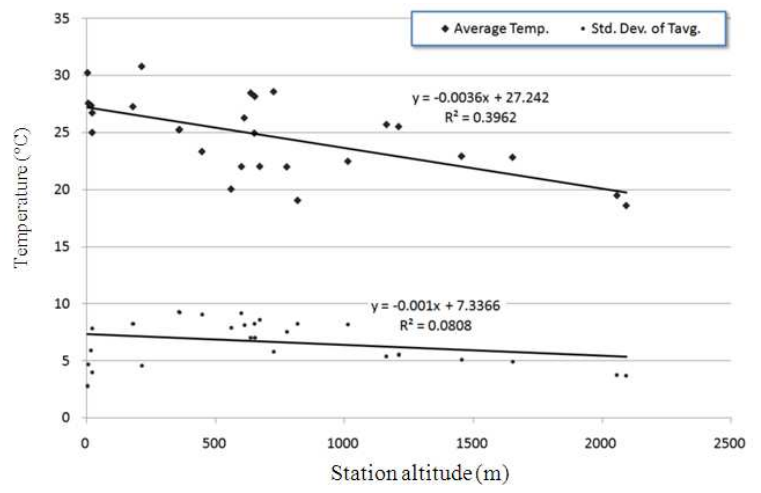

Fig. 3: Average annual temperature values and standard deviation as related to station altitude

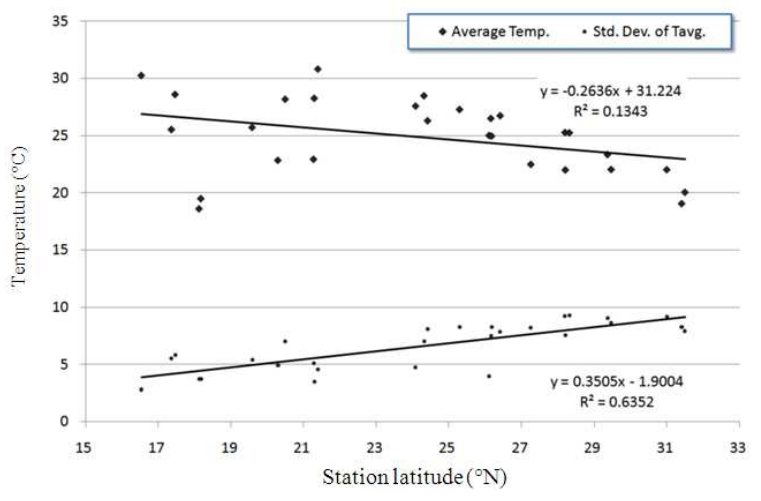

Fig. 4: Average annual temperature values and standard deviation as related to station latitude 
Am. J. Environ. Sci., 6 (2): 191-203, 2010

Table 2: Monthly trends in maximum temperature as estimated from Mann-Kendall and Sen slope statistics for various meteorological station

\begin{tabular}{|c|c|c|c|c|c|c|c|c|c|c|c|c|}
\hline \multirow[b]{2}{*}{ Station } & \multicolumn{10}{|c|}{ Sen's slope (Q) } & \multirow[b]{2}{*}{11} & \multirow[b]{2}{*}{12} \\
\hline & 1 & 2 & 3 & 4 & 5 & 6 & 7 & 8 & 9 & 10 & & \\
\hline TURAIF & 0.02 & 0.09 & 0.12 & 0.04 & $0.07^{*}$ & $0.06^{*}$ & 0.05 & $0.10^{n}$ & 0.01 & 0.07 & 0.03 & 0.02 \\
\hline ARAR & 0.06 & $0.11^{*}$ & $0.16^{*}$ & $0.09^{*}$ & $0.11^{*}$ & $0.12^{*}$ & $0.09^{*}$ & $0.13^{*}$ & $0.06^{*}$ & $0.13^{*}$ & 0.04 & 0.10 \\
\hline AL JOUF & 0.00 & 0.07 & 0.11 & $0.05^{*}$ & 0.05 & $0.06^{*}$ & 0.05 & $0.10^{*}$ & 0.03 & $0.09^{*}$ & 0.05 & 0.01 \\
\hline RAFHA & -0.03 & 0.06 & $0.12^{*}$ & $0.05^{*}$ & 0.04 & $0.05^{*}$ & 0.01 & $0.09^{*}$ & 0.00 & $0.08^{*}$ & 0.01 & -0.02 \\
\hline QAISUMAH & -0.01 & $0.09^{*}$ & $0.13^{*}$ & $0.08^{*}$ & $0.08^{*}$ & $0.08^{*}$ & 0.05 & $0.11^{*}$ & $0.05^{*}$ & $0.12^{*}$ & 0.02 & 0.01 \\
\hline TABUK & -0.01 & 0.07 & 0.05 & 0.03 & 0.02 & 0.02 & 0.02 & $0.05^{*}$ & 0.00 & 0.06 & 0.05 & 0.00 \\
\hline HAFR AL-BATIN & -0.07 & 0.06 & $0.18^{*}$ & 0.04 & 0.03 & -0.01 & 0.04 & 0.10 & 0.04 & 0.09 & -0.09 & -0.10 \\
\hline HAIL & 0.01 & $0.11^{*}$ & $0.11^{*}$ & $0.11^{*}$ & $0.08^{*}$ & $0.10^{*}$ & $0.07^{*}$ & $0.13^{*}$ & $0.07^{*}$ & $0.10^{*}$ & 0.03 & 0.10 \\
\hline WEJH & 0.00 & 0.06 & 0.05 & 0.00 & 0.02 & 0.02 & $0.06^{*}$ & 0.03 & $0.05^{*}$ & 0.03 & 0.05 & 0.01 \\
\hline GASSIM & 0.04 & $0.16^{*}$ & $0.18^{*}$ & $0.17^{*}$ & $0.14^{*}$ & $0.14^{*}$ & $0.10^{*}$ & $0.16^{*}$ & $0.11^{*}$ & $0.14^{*}$ & 0.07 & 0.09 \\
\hline DHAHRAN & 0.04 & $0.11^{*}$ & $0.17^{*}$ & $0.10^{*}$ & $0.12^{*}$ & $0.10^{*}$ & $0.07^{*}$ & $0.10^{*}$ & 0.05 & $0.12^{*}$ & 0.05 & 0.06 \\
\hline AHSA & 0.02 & $0.13^{*}$ & $0.19^{*}$ & $0.15^{*}$ & $0.11^{*}$ & $0.12 *$ & $0.08^{*}$ & $0.10^{*}$ & $0.06^{*}$ & $0.09^{*}$ & 0.02 & 0.12 \\
\hline MADINA & -0.03 & $0.09^{*}$ & $0.11^{*}$ & $0.09^{*}$ & 0.04 & $0.04^{*}$ & 0.03 & $0.07^{*}$ & $0.04^{*}$ & $0.05^{*}$ & 0.04 & 0.07 \\
\hline RIYADH (New) & 0.00 & $0.11^{*}$ & $0.14^{*}$ & $0.14^{*}$ & $0.07^{*}$ & $0.09^{*}$ & 0.06 & $0.07^{*}$ & $0.05^{*}$ & 0.04 & 0.01 & 0.08 \\
\hline YENBO & 0.02 & $0.09^{*}$ & $0.10^{*}$ & $0.09^{*}$ & $0.14 *$ & $0.10^{*}$ & $0.09^{*}$ & $0.13^{*}$ & $0.09^{*}$ & $0.09^{*}$ & 0.10 & $0.09^{*}$ \\
\hline JEDDAH & -0.01 & $0.07^{*}$ & 0.06 & 0.04 & $0.07^{*}$ & $0.06^{*}$ & $0.07^{*}$ & $0.07^{*}$ & $0.05^{*}$ & $0.05^{*}$ & 0.05 & 0.03 \\
\hline MAKKAH & -0.02 & 0.10 & 0.04 & 0.07 & 0.08 & 0.01 . & 0.02 & 0.04 . & 0.04 & 0.04 & 0.03 . & 0.08 \\
\hline TAIF & 0.02 & $0.14^{*}$ & $0.09^{*}$ & $0.09^{*}$ & $0.09^{*}$ & $0.06^{*}$ & 0.05 & $0.07^{*}$ & $0.06^{*}$ & $0.08^{*}$ & $0.06^{*}$ & 0.07 \\
\hline BAHA & 0.02 & $0.12^{*}$ & $0.12^{*}$ & $0.14^{*}$ & $0.12^{*}$ & 0.07 & 0.05 & $0.06^{*}$ & $0.07^{*}$ & $0.04^{*}$ & $0.07^{*}$ & 0.09 \\
\hline W-DAWASIR & 0.01 & $0.13^{*}$ & $0.13^{*}$ & 0.04 & $0.08^{*}$ & $0.04^{*}$ & 0.04 & $0.08^{*}$ & $0.06^{*}$ & $0.06^{*}$ & $0.07^{*}$ & 0.09 \\
\hline BISHA & 0.05 & $0.14^{*}$ & $0.09^{*}$ & $0.09^{*}$ & $0.11^{*}$ & $0.09^{*}$ & $0.07^{*}$ & $0.09^{*}$ & $0.09^{*}$ & $0.08^{*}$ & $0.09^{*}$ & $0.10^{*}$ \\
\hline ABHA & 0.02 & $0.13^{*}$ & $0.10^{*}$ & $0.08^{*}$ & $0.08^{*}$ & 0.03 & 0.03 & $0.04^{*}$ & $0.05^{*}$ & $0.04^{*}$ & $0.04^{*}$ & 0.06 \\
\hline KHAMIS MUSHAIT & 0.05 & $0.15^{*}$ & $0.10^{*}$ & $0.09^{*}$ & $0.10^{*}$ & 0.04 & 0.02 & $0.05^{*}$ & $0.06^{*}$ & $0.04^{*}$ & $0.05^{*}$ & $0.08^{*}$ \\
\hline NEJRAN & $0.10^{*}$ & $0.17^{*}$ & $0.10^{*}$ & $0.12^{*}$ & $0.11^{*}$ & $0.08^{*}$ & $0.08^{*}$ & $0.10^{*}$ & $0.11^{*}$ & $0.12^{*}$ & $0.11^{*}$ & $0.12^{*}$ \\
\hline SHARURRAH & 0.06 & $0.13^{*}$ & 0.04 & $0.08^{*}$ & $0.06^{*}$ & $0.04^{*}$ & 0.04 & 0.01 & $0.04^{*}$ & 0.02 & $0.07^{*}$ & 0.05 \\
\hline GIZAN & 0.01 & 0.03 & 0.01 & 0.00 & -0.01 & -0.01 & -0.01 & 0.00 & -0.01 & -0.03 & 0.01 & 0.00 \\
\hline TURAIF & 0.03 & 0.2 & $0.32^{*}$ & 0.15 & 0.2 & $\begin{array}{r}\text { ndall } \tau \\
0.32^{*}\end{array}$ & 0.17 & $0.33^{*}$ & 0.11 & 0.22 & 0.06 & 0.03 \\
\hline ARAR & 0.17 & $0.30^{*}$ & $0.38^{*}$ & $0.35^{*}$ & $0.45^{*}$ & $0.58^{*}$ & $0.34^{*}$ & $0.45^{*}$ & $0.36^{*}$ & $0.45^{\circ}$ & 0.17 & 0.18 \\
\hline AL JOUF & 0.01 & 0.19 & $0.33^{*}$ & $0.31^{*}$ & 0.23 & $0.38^{*}$ & $0.26^{*}$ & $0.38^{*}$ & 0.23 & $0.39^{*}$ & 0.15 & 0.02 \\
\hline RAFHA & -0.08 & 0.17 & $0.34^{*}$ & $0.26^{*}$ & 0.17 & 0.29 & 0.08 & $0.32^{*}$ & 0.02 & $0.36^{*}$ & 0.03 & -0.04 \\
\hline QAISUMAH & -0.02 & $0.32^{*}$ & $0.35^{*}$ & $0.32^{*}$ & $0.41^{*}$ & $0.44^{*}$ & 0.25 & $0.43^{*}$ & $0.31^{*}$ & $0.44^{*}$ & 0.07 & 0.04 \\
\hline TABUK & -0.02 & 0.20 & 0.12 & 0.15 & 0.07 & 0.12 & 0.11 & $0.27^{*}$ & 0.03 & 0.25 & 0.12 & 0.00 \\
\hline HAFR AL-BATIN & -0.11 & 0.20 & $0.41^{*}$ & 0.09 & 0.05 & -0.05 & 0.13 & 0.24 & 0.12 & 0.19 & -0.18 & -0.14 \\
\hline HAIL & 0.01 & $0.33^{*}$ & $0.38^{*}$ & $0.49^{*}$ & $0.47^{*}$ & $0.47^{*}$ & $0.30^{*}$ & $0.52 *$ & $0.38^{*}$ & $0.49^{*}$ & 0.11 & 0.16 \\
\hline WEJH & 0.01 & 0.21 & 0.13 & 0.00 & 0.09 & 0.13 & $0.47^{*}$ & 0.22 & $0.33^{*}$ & 0.18 & 0.22 & 0.02 \\
\hline GASSIM & 0.12 & $0.42^{*}$ & $0.42^{\circ}$ & $0.52^{*}$ & 0.52 & $0.57^{*}$ & $0.42 *$ & $0.60^{*}$ & $0.57^{*}$ & $0.61^{*}$ & 0.23 & 0.25 \\
\hline DHAHRAN & 0.16 & $0.44^{*}$ & $0.45^{*}$ & $0.33^{*}$ & $0.45^{*}$ & $0.56^{*}$ & $0.37^{*}$ & $0.43^{*}$ & 0.24 & $0.51^{*}$ & 0.21 & 0.18 \\
\hline AHSA & 0.03 & $0.30^{*}$ & $0.51^{*}$ & $0.45^{*}$ & $0.45^{*}$ & $0.61^{*}$ & $0.40^{*}$ & $0.45^{*}$ & $0.45^{*}$ & $0.49^{*}$ & 0.05 & 0.21 \\
\hline MADINA & -0.11 & $0.29^{*}$ & $0.32^{*}$ & $0.37^{*}$ & 0.21 & $0.30^{*}$ & 0.18 & $0.39^{*}$ & $0.36^{*}$ & $0.37^{*}$ & 0.17 & 0.16 \\
\hline RIYADH (New) & 0.00 & $0.32^{*}$ & $0.43^{*}$ & $0.42^{*}$ & $0.35^{*}$ & $0.43^{*}$ & 0.26 & $0.34^{*}$ & $0.38^{*}$ & 0.23 & 0.03 & 0.10 \\
\hline RIYADH (Old) & -0.13 & 0.26 & 0.25 & 0.26 & $0.29^{*}$ & $0.39^{*}$ & 0.11 & $0.39^{*}$ & $0.10^{*}$ & 0.19 & -0.06 & 0.05 \\
\hline YENBO & 0.11 & $0.32^{*}$ & $0.37^{*}$ & $0.37^{*}$ & $0.44^{*}$ & $0.37^{*}$ & $0.35^{*}$ & $0.47^{*}$ & $0.40^{*}$ & $0.49^{*}$ & $0.37^{*}$ & $0.33^{*}$ \\
\hline JEDDAH & -0.03 & $0.30^{*}$ & 0.21 & 0.26 & $0.35^{*}$ & $0.41^{*}$ & $0.42^{*}$ & $0.51^{*}$ & $0.29^{*}$ & $0.33^{*}$ & $0.28^{*}$ & 0.14 \\
\hline МАКKAH & -0.11 & 0.22 & 0.11 & 0.26 & 0.25 & 0.10 & 0.12 & $0.23^{*}$ & 0.18 & 0.20 & 0.08 & 0.18 \\
\hline TAIF & 0.11 & $0.45^{*}$ & $0.39^{*}$ & $0.35^{*}$ & $0.38^{*}$ & $0.35^{*}$ & 0.23 & $0.42^{*}$ & $0.40^{*}$ & $0.40^{*}$ & $0.35^{*}$ & 0.24 \\
\hline BAHA & 0.07 & $0.40^{*}$ & $0.39^{*}$ & $0.38^{*}$ & $0.32^{*}$ & 0.29 & 0.22 & $0.36^{*}$ & $0.38^{*}$ & 0.23 & $0.37^{*}$ & 0.28 \\
\hline W-DAWASIR & 0.05 & $0.36^{*}$ & $0.41^{*}$ & 0.20 & $0.42^{*}$ & $0.31^{*}$ & 0.22 & $0.38^{*}$ & $0.35^{*}$ & $0.34^{*}$ & $0.35^{*}$ & 0.23 \\
\hline BISHA & 0.19 & $0.44^{*}$ & $0.42^{*}$ & $0.35^{*}$ & $0.50^{*}$ & $0.50^{*}$ & $0.49^{*}$ & $0.57^{*}$ & $0.60^{*}$ & $0.45^{*}$ & $0.40^{*}$ & $0.43^{*}$ \\
\hline ABHA & 0.15 & $0.45^{*}$ & $0.38^{*}$ & $0.32^{*}$ & $0.39^{*}$ & 0.24 & 0.22 & $0.38^{*}$ & $0.46^{*}$ & $0.36^{*}$ & $0.27^{*}$ & 0.22 \\
\hline KHAMIS MUSHAIT & 0.19 & $0.46^{*}$ & $0.35^{*}$ & $0.33^{*}$ & $0.44^{*}$ & 0.24 & 0.10 & $0.49^{*}$ & $0.50^{*}$ & $0.30^{*}$ & $0.33^{*}$ & $0.30^{*}$ \\
\hline NEJRAN & $0.33^{*}$ & $0.44^{*}$ & $0.44^{*}$ & $0.36^{*}$ & $0.54^{*}$ & $0.52^{*}$ & $0.44^{*}$ & $0.63^{*}$ & $0.68^{*}$ & $0.57^{*}$ & $0.44^{*}$ & $0.47^{*}$ \\
\hline SHARURRAH & 0.17 & $0.32^{*}$ & 0.24 & $0.30^{*}$ & $0.34^{*}$ & $0.32^{*}$ & 0.22 & 0.12 & $0.34^{*}$ & 0.08 & $0.30^{*}$ & 0.19 \\
\hline GIZAN & 0.09 & 0.18 & 0.09 & 0.01 & -0.04 & -0.12 & -0.15 & -0.04 & -0.12 & -0.16 & 0.07 & 0.00 \\
\hline
\end{tabular}

Symbol's key: *: Significant at 95\%; ": Significant at $99 \%$

Saudi Arabia extends widely from $15^{\circ}$ North to more than $31^{\circ}$ and therefore one expect a wide temperature variation with latitude. The relationship between $\mathrm{Ta}$ and its standard deviation and stations latitude was plotted in Fig. 4. A weak and negative linear relationship was found between Ta and latitude with a slope of -0.26 and $\mathrm{R}^{2}=0.13$. Therefore, temperature seemed to be higher in the northern part of SA compared to southern parts. This is an expected result because northern parts are prevailed by desert climate with high average Ty and high temperature variations as well. Indeed, temperature variations, as indicated by standard deviation, seemed to increase steadily with in the northern parts, large altitudes, at a rate of $0.35^{\circ} \mathrm{C}$ and $\mathrm{R}^{2}=0.63$, shown in Fig. 4 .

Trend analyses for the time series data of monthly temperatures were performed using Kendall and Sen's statistics. The values of Kendall $\tau$ and Sen's $Q$ are shown in Table 2-4 for maximum, average and minimum temperatures respectively. In such tables, the significance degree is marked beside each value. 
Am. J. Environ. Sci., 6 (2): 191-203, 2010

Table 3: Monthly trends in average temperature as estimated from Mann-Kendall and Sen slope statistics for various meteorological station

\begin{tabular}{|c|c|c|c|c|c|c|c|c|c|c|c|c|}
\hline \multirow[b]{2}{*}{ Station } & \multicolumn{10}{|c|}{ Sen's slope $(\mathbf{Q})$} & \multirow[b]{2}{*}{11} & \multirow[b]{2}{*}{12} \\
\hline & 1 & 2 & 3 & 4 & 5 & 6 & 7 & 8 & 9 & 10 & & \\
\hline TURAIF & -0.01 & 0.05 & 0.07 & 0.03 & 0.06 & $0.05^{*}$ & 0.06 & 0.08 & 0.02 & 0.05 & -0.01 & -0.01 \\
\hline ARAR & 0.02 & 0.07 & $0.12^{*}$ & $0.07^{*}$ & $0.08^{*}$ & $0.08^{*}$ & $0.09^{*}$ & $0.11^{*}$ & $0.06^{*}$ & $0.10^{*}$ & 0.04 & 0.08 \\
\hline AL JOUF & 0.00 & 0.05 & 0.07 & 0.05 & $0.07^{*}$ & 0.06 & 0.06 & $0.09^{*}$ & 0.03 & $0.08^{*}$ & 0.06 & 0.01 \\
\hline RAFHA & -0.01 & 0.04 & 0.08 & 0.04 & 0.05 & $0.05^{*}$ & 0.03 & $0.10^{*}$ & 0.02 & $0.07^{*}$ & -0.02 & 0.00 \\
\hline QAISUMAH & 0.00 & 0.07 & $0.09^{*}$ & 0.06 & $0.08^{*}$ & $0.08^{*}$ & 0.05 & $0.10^{*}$ & $0.05^{*}$ & $0.08^{*}$ & -0.01 & 0.02 \\
\hline TABUK & 0.02 & 0.06 & 0.04 & 0.03 & 0.04 & $0.04^{*}$ & $0.04^{*}$ & $0.07^{*}$ & $0.04^{*}$ & $0.06^{*}$ & 0.03 & -0.01 \\
\hline HAFR AL-BATIN & -0.02 & 0.06 & $0.18^{*}$ & 0.01 & 0.08 & 0.04 & 0.01 & 0.06 & 0.04 & 0.05 & -0.02 & -0.05 \\
\hline HAIL & 0.00 & $0.08^{*}$ & $0.07^{*}$ & $0.07^{*}$ & $0.06^{*}$ & $0.09^{*}$ & $0.06^{*}$ & $0.10^{*}$ & $0.06^{*}$ & $0.07^{*}$ & 0.01 & 0.05 \\
\hline WEJH & 0.00 & 0.04 & 0.03 & 0.02 & 0.03 & 0.02 & $0.05^{*}$ & $0.04^{*}$ & $0.04^{*}$ & 0.02 & 0.04 & 0.00 \\
\hline GASSIM & 0.00 & $0.08^{*}$ & $0.09^{*}$ & $0.10^{*}$ & $0.07^{*}$ & $0.08^{*}$ & $0.05^{*}$ & $0.10^{*}$ & $0.06^{*}$ & $0.06^{*}$ & 0.03 & 0.07 \\
\hline DHAHRAN & 0.02 & $0.07^{*}$ & $0.09^{*}$ & $0.06^{*}$ & $0.09^{*}$ & $0.07^{*}$ & $0.06^{*}$ & $0.08^{*}$ & $0.05^{*}$ & 0.08 & 0.01 & 0.04 \\
\hline AHSA & -0.05 & 0.03 & $0.09^{*}$ & $0.09^{*}$ & 0.09 & 0.07 & 0.04 & 0.05 & 0.03 & $0.04^{*}$ & -0.03 & 0.01 \\
\hline MADINA & -0.03 & 0.08 & $0.08^{*}$ & $0.08^{*}$ & 0.03 & $0.03^{*}$ & 0.03 & $0.07^{*}$ & $0.04^{*}$ & $0.04^{*}$ & 0.03 & 0.05 \\
\hline RIYADH (New) & 0.02 & 0.08 & 0.10 & $0.12^{*}$ & $0.08^{*}$ & $0.08^{*}$ & 0.06 & $0.06^{*}$ & $0.05^{*}$ & 0.01 & 0.00 & 0.04 \\
\hline RIYADH (Old) & 0.00 & $0.07^{*}$ & $0.08^{*}$ & 0.07 & $0.07^{*}$ & $0.07^{*}$ & $0.04^{*}$ & $0.09^{*}$ & $0.04^{*}$ & $0.05^{*}$ & 0.00 & 0.04 \\
\hline YENBO & 0.01 & 0.07 & 0.05 & 0.06 & $0.10^{*}$ & $0.08^{*}$ & $0.09^{*}$ & $0.12^{*}$ & $0.09^{*}$ & $0.07^{*}$ & 0.05 & $0.04^{*}$ \\
\hline JEDDAH & -0.02 & 0.06 & 0.02 & 0.03 & 0.02 & $0.04^{*}$ & $0.06^{*}$ & $0.07^{*}$ & $0.04^{*}$ & $0.02^{*}$ & $0.03^{*}$ & 0.02 \\
\hline MAKKAH & -0.02 & 0.09 & 0.04 & 0.06 & 0.05 & 0.03 & 0.04 & $0.04^{*}$ & 0.04 & $0.04^{*}$ & 0.02 & 0.05 \\
\hline TAIF & -0.01 & $0.07^{*}$ & 0.04 & 0.04 & 0.03 & 0.03 & 0.01 & $0.03^{*}$ & 0.02 & 0.02 & 0.02 & 0.03 \\
\hline BAHA & 0.02 & 0.10 & 0.06 & $0.11^{*}$ & 0.07 & 0.03 & 0.03 & 0.03 & $0.06^{*}$ & 0.03 & $0.04^{*}$ & 0.05 \\
\hline W-DAWASIR & -0.01 & 0.07 & 0.03 & -0.03 & 0.02 & 0.01 & 0.00 & 0.04 & 0.04 & $0.02^{*}$ & $0.07^{*}$ & 0.06 \\
\hline BISHA & 0.01 & $0.08^{*}$ & 0.04 & 0.07 & $0.08^{*}$ & $0.07^{*}$ & $0.06^{*}$ & $0.06^{*}$ & $0.06^{*}$ & $0.04^{*}$ & 0.05 & $0.05^{\circ}$ \\
\hline ABHA & 0.03 & $0.07^{*}$ & $0.05^{*}$ & $0.07^{*}$ & $0.07^{*}$ & $0.03^{*}$ & 0.02 & $0.04^{*}$ & $0.04^{*}$ & $0.04^{*}$ & $0.04^{*}$ & 0.04 \\
\hline KHAMIS MUSHAIT & $0.06^{\circ}$ & $0.10^{*}$ & $0.07^{*}$ & $0.08^{*}$ & $0.09^{*}$ & $0.07^{*}$ & $0.04^{*}$ & $0.07^{*}$ & $0.08^{*}$ & $0.08^{*}$ & $0.10^{*}$ & $0.09^{*}$ \\
\hline NEJRAN & 0.03 & 0.08 & 0.02 & $0.06^{*}$ & $0.06^{*}$ & 0.06 & $0.06^{*}$ & $0.06^{*}$ & $0.08^{*}$ & $0.08^{*}$ & 0.07 & $0.07^{*}$ \\
\hline SHARURRAH & 0.03 & 0.06 & -0.01 & 0.05 & 0.03 & 0.04 & 0.01 & -0.01 & $0.03^{*}$ & -0.01 & $0.02^{*}$ & 0.00 \\
\hline GIZAN & $0.04^{*}$ & $0.04^{*}$ & 0.02 & $0.03^{*}$ & $0.02^{*}$ & 0.02 & 0.02 & $0.02^{*}$ & $0.04^{*}$ & $0.02^{*}$ & $0.05^{*}$ & 0.00 \\
\hline \multicolumn{13}{|c|}{ Kendall $\tau$} \\
\hline TURAIF & -0.03 & 0.16 & 0.20 & 0.10 & 0.25 & $0.33^{*}$ & 0.21 & 0.25 & 0.12 & 0.17 & -0.02 & -0.01 \\
\hline ARAR & 0.08 & 0.25 & $0.32^{*}$ & $0.27^{*}$ & $0.37^{*}$ & $0.54^{*}$ & $0.35^{*}$ & $0.43^{*}$ & $0.36^{*}$ & $0.42^{*}$ & 0.09 & 0.21 \\
\hline AL JOUF & 0.00 & 0.26 & 0.25 & 0.23 & $0.26^{*}$ & 0.21 & 0.24 & $0.30^{*}$ & 0.17 & $0.36^{*}$ & 0.13 & 0.04 \\
\hline RAFHA & -0.02 & 0.14 & 0.22 & 0.18 & 0.22 & $0.41^{*}$ & 0.17 & $0.36^{*}$ & 0.11 & $0.30^{*}$ & -0.06 & 0.01 \\
\hline QAISUMAH & 0.01 & 0.26 & $0.28^{*}$ & 0.25 & $0.38^{*}$ & $0.53^{*}$ & 0.26 & $0.41^{*}$ & $0.27^{*}$ & $0.41^{*}$ & -0.07 & 0.05 \\
\hline TABUK & 0.05 & 0.20 & 0.15 & 0.18 & 0.19 & $0.36^{*}$ & $0.26^{*}$ & $0.38^{*}$ & $0.28^{*}$ & $0.33^{*}$ & 0.10 & -0.03 \\
\hline HAFR AL-BATIN & -0.06 & 0.17 & $0.46^{*}$ & 0.05 & 0.16 & 0.22 & 0.04 & 0.18 & 0.22 & 0.11 & -0.06 & -0.03 \\
\hline HAIL & 0.01 & $0.26^{*}$ & $0.29^{*}$ & $0.40^{*}$ & $0.32^{*}$ & $0.53^{*}$ & $0.33^{*}$ & $0.46^{*}$ & $0.37^{*}$ & $0.42^{*}$ & 0.03 & 0.21 \\
\hline WEJH & -0.01 & 0.21 & 0.14 & 0.12 & 0.15 & 0.18 & $0.43^{*}$ & $0.37^{*}$ & $0.33^{*}$ & 0.23 & 0.20 & 0.02 \\
\hline GASSIM & -0.01 & $0.27^{*}$ & $0.30^{*}$ & $0.39^{*}$ & $0.35^{*}$ & $0.58^{*}$ & $0.29^{*}$ & $0.48^{*}$ & $0.47^{*}$ & $0.35^{*}$ & 0.15 & 0.17 \\
\hline DHAHRAN & 0.09 & $0.29^{*}$ & $0.34^{*}$ & $0.31^{*}$ & $0.41^{*}$ & $0.52^{*}$ & $0.50^{*}$ & $0.59^{*}$ & $0.38^{*}$ & $0.43^{*}$ & 0.10 & 0.18 \\
\hline AHSA & -0.09 & 0.11 & $0.36^{*}$ & $0.34^{*}$ & 0.21 & 0.29 & 0.17 & 0.15 & 0.15 & 0.16 & -0.08 & 0.12 \\
\hline MADINA & -0.11 & 0.24 & $0.27^{*}$ & $0.35^{*}$ & 0.11 & $0.30^{*}$ & 0.22 & $0.37^{*}$ & $0.33^{*}$ & $0.36^{*}$ & 0.19 & 0.16 \\
\hline RIYADH (New) & 0.09 & 0.26 & $0.48^{*}$ & $0.43^{*}$ & $0.41^{*}$ & $0.57^{*}$ & 0.28 & $0.30^{*}$ & $0.33^{*}$ & 0.05 & 0.02 & 0.09 \\
\hline RIYADH (Old) & -0.01 & $0.28^{*}$ & 0.27 & 0.24 & $0.40^{*}$ & $0.49^{*}$ & 0.28 & $0.43^{*}$ & $0.38^{*}$ & $0.34^{*}$ & -0.01 & 0.12 \\
\hline YENBO & 0.04 & 0.24 & 0.13 & $0.30^{*}$ & $0.35^{*}$ & $0.46^{*}$ & $0.48^{*}$ & 0.54 & $0.49^{*}$ & $0.57^{*}$ & 0.23 & 0.20 \\
\hline JEDDAH & -0.16 & 0.25 & 0.07 & 0.17 & 0.17 & $0.36^{*}$ & $0.39^{*}$ & $0.47^{*}$ & $0.38^{*}$ & $0.29^{*}$ & 0.23 & 0.14 \\
\hline MAKKAH & -0.10 & 0.28 & 0.11 & 0.26 & 0.24 & 0.26 & 0.25 & $0.46^{*}$ & 0.22 & $0.29^{*}$ & 0.19 & 0.22 \\
\hline TAIF & -0.02 & $0.33^{*}$ & $0.18^{*}$ & 0.18 & 0.15 & 0.20 & 0.06 & $0.36^{*}$ & 0.21 & 0.23 & 0.19 & 0.12 \\
\hline BAHA & 0.09 & $0.41^{*}$ & 0.32 & $0.43^{*}$ & 0.20 & 0.20 & 0.18 & 0.15 & $0.32^{*}$ & 0.18 & 0.19 & 0.25 \\
\hline W-DAWASIR & -0.07 & 0.21 & 0.07 & -0.08 & 0.07 & 0.05 & 0.01 & 0.19 & 0.18 & 0.09 & 0.23 & 0.22 \\
\hline BISHA & 0.07 & $0.30^{*}$ & 0.19 & $0.27^{*}$ & $0.38^{*}$ & $0.50^{*}$ & $0.40^{\circ}$ & $0.45^{*}$ & $0.42^{*}$ & 0.24 & 0.23 & 0.25 \\
\hline ABHA & 0.16 & $0.49^{*}$ & $0.36^{*}$ & 0.29 & $0.39^{*}$ & $0.31^{*}$ & 0.20 & $0.42^{*}$ & $0.39^{*}$ & $0.38^{*}$ & $0.29^{*}$ & $0.42^{*}$ \\
\hline KHAMIS MUSHAIT & $0.46^{*}$ & $0.56^{*}$ & $0.45^{*}$ & $0.42^{*}$ & $0.48^{*}$ & $0.49^{*}$ & $0.36^{*}$ & $0.60^{*}$ & $0.63^{*}$ & $0.58^{*}$ & $0.56^{*}$ & $0.60^{\circ}$ \\
\hline NEJRAN & 0.09 & 0.25 & 0.11 & $0.29^{*}$ & $0.31^{*}$ & 0.25 & $0.44^{*}$ & $0.37^{*}$ & $0.44^{*}$ & $0.37^{*}$ & 0.26 & $0.31^{*}$ \\
\hline SHARURRAH & 0.09 & 0.12 & -0.04 & 0.17 & 0.16 & 0.21 & 0.08 & -0.04 & 0.21 & -0.06 & 0.12 & -0.02 \\
\hline GIZAN & $0.33^{*}$ & $0.28^{*}$ & 0.19 & $0.28^{*}$ & $0.29^{*}$ & 0.25 & 0.24 & $0.35^{*}$ & $0.46^{*}$ & $0.28^{*}$ & $0.41^{*}$ & 0.00 \\
\hline
\end{tabular}

Symbol's key: *: Significant at 95\%; ": Significant at $99 \%$ 
Am. J. Environ. Sci., 6 (2): 191-203, 2010

Table 4: Monthly trends in minimum temperature as estimated from Mann-Kendall and Sen slope statistics for various meteorological station

\begin{tabular}{|c|c|c|c|c|c|c|c|c|c|c|c|c|}
\hline \multirow[b]{2}{*}{ Station } & \multicolumn{10}{|c|}{ Sen's slope $(Q)$} & \multirow[b]{2}{*}{11} & \multirow[b]{2}{*}{12} \\
\hline & 1 & $2^{*}$ & 3 & 4 & 5 & 6 & 7 & 8 & 9 & 10 & & \\
\hline TURAIF & -0.02 & 0.03 & 0.04 & 0.02 & 0.05 & $0.06^{*}$ & $0.07^{*}$ & $0.08^{*}$ & $0.06^{*}$ & 0.06 & -0.02 & -0.02 \\
\hline ARAR & 0.00 & 0.04 & 0.06 & 0.04 & $0.07^{*}$ & $0.06^{*}$ & $0.05^{*}$ & $0.10^{*}$ & 0.03 & $0.09^{*}$ & -0.01 & 0.03 \\
\hline AL JOUF & 0.04 & $0.09^{*}$ & 0.07 & $0.06^{*}$ & $0.09^{*}$ & $0.07^{*}$ & $0.08^{*}$ & $0.10^{*}$ & $0.07^{*}$ & $0.11^{*}$ & 0.04 & 0.03 \\
\hline RAFHA & 0.00 & 0.01 & 0.04 & 0.04 & 0.05 & $0.10^{*}$ & $0.08^{*}$ & $0.12^{*}$ & $0.06^{*}$ & $0.09^{*}$ & -0.02 & 0.02 \\
\hline QAISUMAH & 0.00 & 0.06 & 0.04 & 0.03 & $0.06^{*}$ & $0.09^{*}$ & $0.06^{*}$ & $0.12^{*}$ & $0.05^{*}$ & $0.06^{*}$ & -0.01 & 0.03 \\
\hline TABUK & 0.01 & 0.04 & 0.04 & 0.03 & 0.05 & $0.05^{*}$ & $0.06^{*}$ & $0.08^{*}$ & $0.06^{*}$ & $0.06^{*}$ & -0.01 & -0.02 \\
\hline HAFR AL-BATIN & 0.03 & $0.11^{*}$ & $0.17^{*}$ & 0.13 & $0.14^{*}$ & $0.10^{*}$ & 0.07 & $0.13^{*}$ & 0.10 & 0.03 & 0.03 & 0.01 \\
\hline HAIL & 0.01 & 0.06 & 0.04 & 0.05 & 0.05 & $0.09^{*}$ & $0.06^{*}$ & $0.14^{*}$ & $0.11^{*}$ & 0.06 & -0.01 & 0.07 \\
\hline WEJH & -0.01 & 0.04 & 0.03 & 0.02 & 0.04 & $0.01^{*}$ & $0.04^{*}$ & $0.05^{*}$ & $0.04^{*}$ & $0.04^{*}$ & 0.03 & 0.00 \\
\hline GASSIM & -0.03 & 0.03 & -0.01 & 0.05 & 0.01 & 0.04 & 0.01 & $0.08^{*}$ & 0.04 & 0.01 & 0.01 & 0.02 \\
\hline DHAHRAN & 0.02 & 0.05 & 0.03 & 0.03 & $0.05^{*}$ & $0.03^{*}$ & $0.04^{*}$ & $0.06^{*}$ & 0.03 & 0.05 & 0.00 & 0.03 \\
\hline AHSA & 0.05 & 0.08 & $0.08^{*}$ & 0.08 & $0.09^{*}$ & 0.13 & $0.06^{*}$ & $0.10^{*}$ & $0.09^{*}$ & $0.08^{*}$ & 0.08 & 0.06 \\
\hline MADINA & 0.00 & 0.06 & 0.03 & $0.05^{*}$ & 0.02 & $0.04^{*}$ & 0.04 & $0.09^{*}$ & $0.07^{*}$ & $0.03^{*}$ & $0.03^{*}$ & 0.06 \\
\hline RIYADH (New) & 0.07 & 0.10 & 0.06 & $0.11^{*}$ & $0.09^{*}$ & $0.11^{*}$ & $0.09^{*}$ & $0.10^{*}$ & $0.11^{*}$ & 0.06 & 0.07 & 0.08 \\
\hline RIYADH (Old) & 0.01 & 0.06 & 0.06 & 0.06 & $0.07^{*}$ & $0.08^{*}$ & $0.05^{*}$ & $0.08^{*}$ & $0.05^{*}$ & 0.04 & 0.02 & 0.05 \\
\hline YENBO & 0.05 & $0.09^{*}$ & 0.01 & 0.03 & 0.05 & 0.03 & $0.07^{*}$ & $0.12^{*}$ & $0.07^{*}$ & 0.03 & 0.04 & 0.05 \\
\hline JEDDAH & 0.00 & 0.07 & 0.01 & 0.01 & 0.00 & 0.05 & $0.07^{*}$ & $0.09^{*}$ & $0.05^{*}$ & 0.02 & 0.02 & 0.00 \\
\hline MAKKAH & 0.03 & $0.13^{*}$ & $0.08^{*}$ & $0.09^{*}$ & 0.06 & $0.10^{*}$ & $0.10^{*}$ & $0.11^{*}$ & $0.08^{*}$ & $0.08^{*}$ & 0.07 & $0.07^{*}$ \\
\hline TAIF & -0.02 & 0.03 & -0.04 & 0.00 & -0.03 & 0.00 & -0.01 & 0.00 & -0.04 & -0.04 & -0.02 & -0.01 \\
\hline BAHA & 0.02 & 0.09 & 0.00 & 0.07 & 0.02 & 0.00 & 0.01 & $0.03^{*}$ & 0.01 & 0.00 & 0.01 & 0.03 \\
\hline W-DAWASIR & 0.00 & 0.06 & -0.01 & $0.01^{*}$ & 0.05 & 0.06 & $0.07^{*}$ & $0.11^{*}$ & $0.10^{*}$ & $0.10^{*}$ & $0.13^{*}$ & $0.11^{*}$ \\
\hline BISHA & 0.02 & 0.07 & -0.01 & 0.04 & $0.05^{*}$ & $0.08^{*}$ & $0.09^{*}$ & $0.08^{*}$ & $0.09^{*}$ & 0.07 & 0.05 & 0.09 \\
\hline ABHA & 0.04 & 0.00 & 0.01 & $0.04^{*}$ & $0.07^{*}$ & $0.04^{*}$ & $0.05^{*}$ & $0.04^{*}$ & $0.05^{*}$ & $0.05^{*}$ & $0.05^{*}$ & 0.04 \\
\hline KHAMIS MUSHAIT & $0.09^{*}$ & 0.03 & 0.04 & $0.07^{*}$ & $0.10^{*}$ & $0.06^{*}$ & 0.06 & $0.08^{*}$ & $0.09^{*}$ & $0.09^{*}$ & $0.13^{*}$ & $0.10^{*}$ \\
\hline NEJRAN & 0.01 & 0.01 & -0.05 & 0.00 & -0.01 & 0.03 & $0.05^{*}$ & 0.00 & $0.05^{*}$ & 0.04 & 0.04 & 0.07 \\
\hline SHARURRAH & 0.02 & -0.04 & -0.06 & 0.00 & 0.05 & $0.07^{*}$ & 0.04 & -0.01 & 0.03 & 0.02 & 0.04 & 0.05 \\
\hline GIZAN & 0.04 & 0.04 & 0.01 & $0.04^{*}$ & $0.04^{*}$ & $0.03^{*}$ & $0.04^{*}$ & 0.03 & $0.06^{*}$ & $0.06^{*}$ & $0.05^{*}$ & 0.00 \\
\hline \multicolumn{13}{|c|}{ Kendall $\tau$} \\
\hline TURAIF & -0.10 & 0.17 & 0.21 & 0.10 & 0.25 & $0.59^{*}$ & $0.35^{*}$ & $0.40^{*}$ & $0.32^{*}$ & 0.23 & -0.08 & -0.03 \\
\hline ARAR & -0.01 & 0.19 & 0.20 & 0.21 & $0.31^{*}$ & $0.52^{*}$ & $0.28^{*}$ & $0.34^{*}$ & 0.26 & $0.34^{*}$ & -0.02 & 0.09 \\
\hline AL JOUF & 0.14 & $0.36^{*}$ & 0.26 & $0.30^{*}$ & $0.42^{*}$ & $0.55^{*}$ & $0.44^{*}$ & $0.45^{*}$ & $0.30^{*}$ & $0.43^{*}$ & 0.18 & 0.13 \\
\hline RAFHA & 0.01 & 0.08 & 0.13 & 0.20 & 0.22 & $0.58^{*}$ & $0.37^{*}$ & $0.39^{*}$ & $0.32^{*}$ & $0.28^{*}$ & -0.04 & 0.04 \\
\hline QAISUMAH & -0.01 & 0.23 & 0.17 & 0.24 & $0.30^{*}$ & $0.53^{*}$ & $0.36^{*}$ & $0.38^{*}$ & $0.30^{*}$ & $0.32^{*}$ & -0.05 & 0.10 \\
\hline TABUK & 0.06 & 0.17 & 0.10 & 0.11 & 0.25 & $0.45^{*}$ & $0.32^{*}$ & $0.40^{*}$ & 0.45 & $0.27^{*}$ & -0.04 & -0.07 \\
\hline HAFR AL-BATIN & 0.06 & $0.37^{*}$ & $0.38^{*}$ & 0.23 & $0.47^{*}$ & $0.35^{*}$ & 0.26 & 0.37 & 0.31 & 0.08 & 0.10 & 0.03 \\
\hline HAIL & 0.04 & 0.17 & 0.16 & 0.26 & 0.23 & $0.44^{*}$ & 0.33 & $0.42^{*}$ & $0.44^{*}$ & 0.22 & -0.05 & 0.19 \\
\hline WEJH & -0.05 & 0.14 & 0.08 & 0.10 & 0.18 & 0.16 & $0.32^{*}$ & $0.36^{*}$ & $0.34^{*}$ & $0.31^{*}$ & 0.12 & 0.03 \\
\hline GASSIM & -0.19 & 0.10 & -0.05 & 0.19 & 0.03 & 0.26 & 0.06 & $0.32^{*}$ & 0.21 & 0.07 & 0.04 & 0.11 \\
\hline DHAHRAN & 0.06 & 0.20 & 0.12 & 0.23 & $0.32^{*}$ & 0.22 & $0.34^{*}$ & $0.40^{*}$ & 0.22 & 0.21 & -0.01 & 0.14 \\
\hline AHSA & 0.23 & 0.29 & $0.33^{*}$ & 0.26 & $0.39^{*}$ & $0.62^{*}$ & $0.30^{*}$ & $0.54^{*}$ & $0.38^{*}$ & $0.43^{*}$ & 0.27 & 0.19 \\
\hline MADINA & -0.03 & 0.25 & 0.11 & $0.31^{*}$ & 0.14 & $0.37^{*}$ & 0.26 & $0.35^{*}$ & $0.36^{*}$ & $0.30^{*}$ & $0.27^{*}$ & 0.20 \\
\hline RIYADH (New) & 0.23 & 0.28 & 0.27 & $0.47^{*}$ & $0.48^{*}$ & $0.64^{*}$ & $0.41^{*}$ & $0.35^{*}$ & $0.47^{*}$ & 0.23 & 0.19 & 0.21 \\
\hline RIYADH (Old) & 0.03 & 0.24 & 0.22 & 0.25 & $0.44^{*}$ & $0.43^{*}$ & $0.35^{*}$ & $0.40^{*}$ & $0.33^{*}$ & 0.17 & 0.10 & 0.19 \\
\hline YENBO & 0.17 & $0.30^{*}$ & 0.02 & 0.12 & 0.23 & 0.24 & 0.48 & $0.52^{*}$ & $0.45^{*}$ & 0.23 & 0.14 & 0.20 \\
\hline JEDDAH & -0.03 & 0.18 & 0.04 & 0.07 & -0.03 & 0.26 & $0.38^{*}$ & $0.41^{*}$ & $0.33^{*}$ & 0.23 & 0.15 & 0.01 \\
\hline MAKKAH & 0.14 & $0.46^{*}$ & $0.36^{*}$ & $0.38^{*}$ & 0.22 & $0.57^{*}$ & $0.55^{*}$ & $0.54^{*}$ & $0.50^{*}$ & $0.54^{*}$ & $0.47^{*}$ & $0.38^{*}$ \\
\hline TAIF & -0.11 & 0.16 & -0.19 & -0.01 & -0.18 & 0.04 & -0.03 & 0.01 & -0.20 & -0.20 & -0.10 & -0.05 \\
\hline BAHA & 0.10 & 0.28 & 0.01 & $0.37^{*}$ & 0.14 & 0.01 & 0.10 & $0.29^{*}$ & 0.13 & -0.02 & 0.08 & 0.09 \\
\hline W-DAWASIR & 0.01 & 0.14 & -0.04 & 0.07 & 0.23 & 0.22 & $0.33^{*}$ & $0.38^{*}$ & $0.41^{*}$ & $0.38^{*}$ & $0.32^{*}$ & $0.33^{*}$ \\
\hline BISHA & 0.07 & 0.25 & -0.04 & 0.17 & $0.34^{*}$ & $0.45^{*}$ & $0.42^{*}$ & $0.39^{*}$ & $0.48^{*}$ & $0.26^{*}$ & 0.19 & 0.25 \\
\hline $\mathrm{ABHA}$ & 0.17 & 0.02 & 0.05 & $0.30^{*}$ & $0.46^{*}$ & $0.36^{*}$ & $0.37^{*}$ & $0.39^{*}$ & $0.46^{*}$ & $0.34^{*}$ & $0.28^{*}$ & 0.18 \\
\hline KHAMIS MUSHAIT & $0.41^{*}$ & 0.15 & 0.23 & $0.40^{*}$ & $0.50^{*}$ & $0.32^{*}$ & 0.23 & $0.36^{*}$ & $0.34^{*}$ & $0.37^{*}$ & $0.50^{*}$ & $0.36^{*}$ \\
\hline NEJRAN & 0.02 & 0.03 & -0.14 & -0.01 & -0.04 & 0.12 & $0.35^{*}$ & 0.01 & $0.28^{*}$ & 0.21 & 0.10 & 0.20 \\
\hline SHARURRAH & 0.04 & -0.05 & -0.16 & -0.01 & 0.24 & $0.30^{*}$ & 0.15 & -0.03 & 0.12 & $0.05^{*}$ & 0.12 & 0.14 \\
\hline GIZAN & 0.20 & 0.23 & 0.06 & $0.31^{*}$ & $0.30^{*}$ & $0.31^{*}$ & $0.31^{*}$ & 0.24 & $0.59^{*}$ & $0.47^{*}$ & $0.38^{*}$ & 0.00 \\
\hline
\end{tabular}

Symbol's key: ": Significant at 95\%; ": Significant at $99 \%$ 


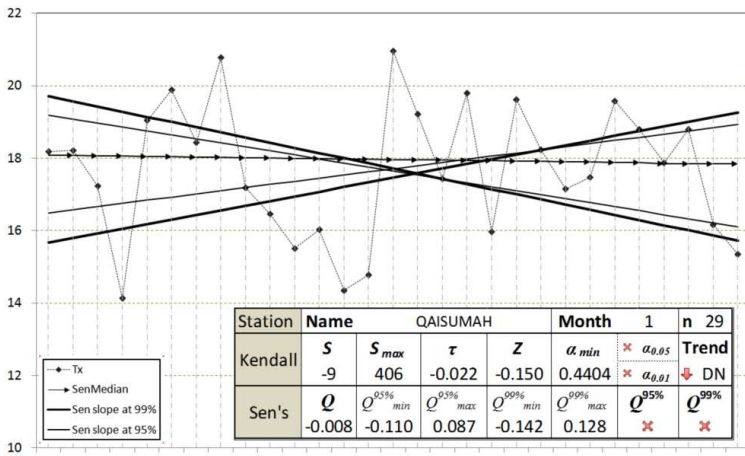

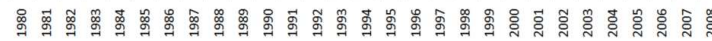

(a)

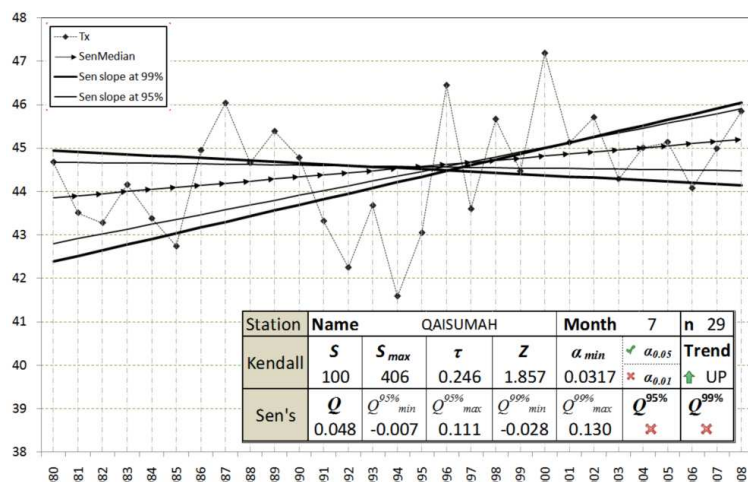

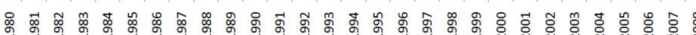
(c)

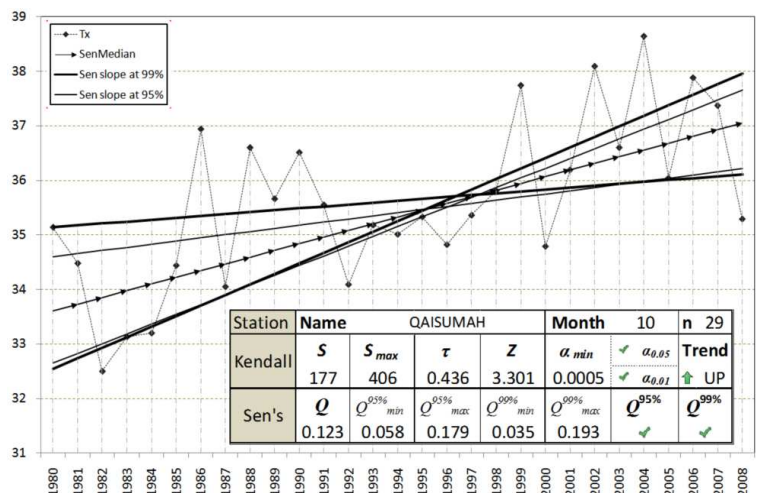

(e)

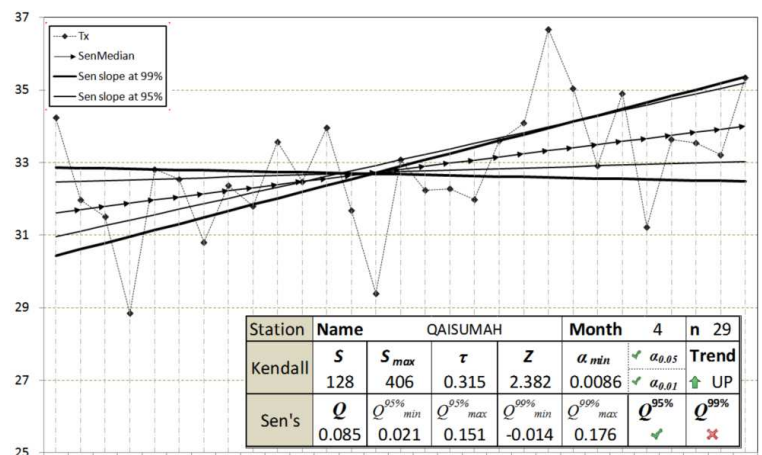

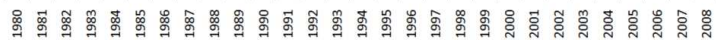

(b)

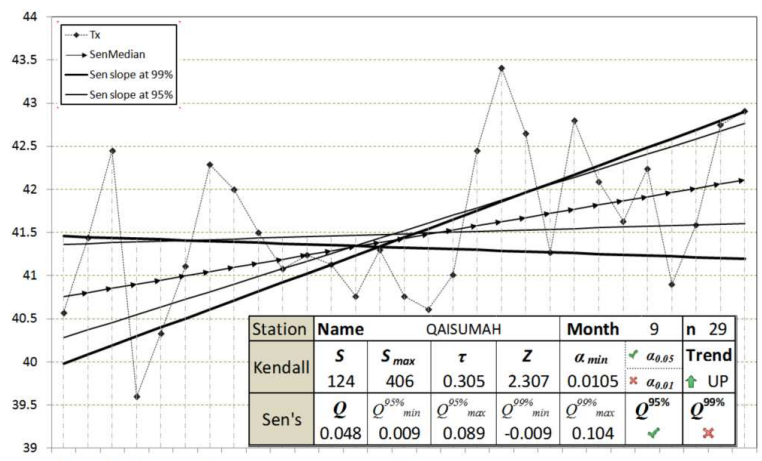

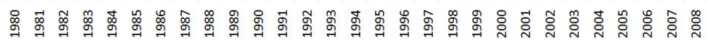

(d)

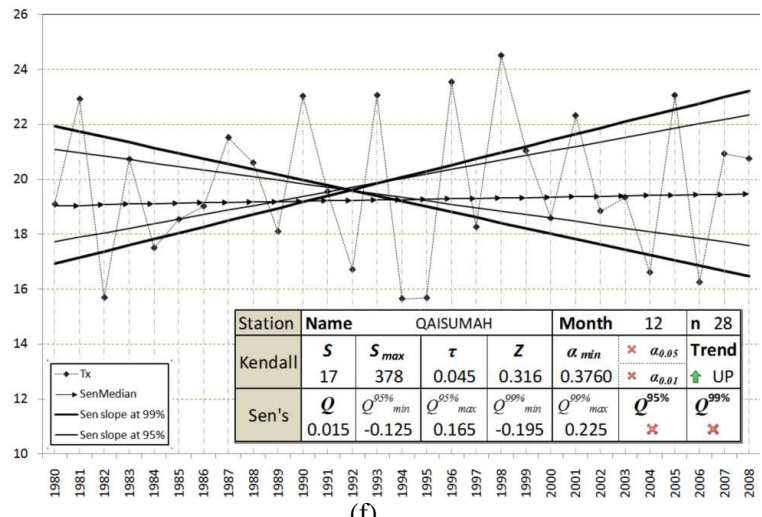

(f)

Fig. 5: Analysis of maximum temperature trends using Mann-Kendall and Sen slope estimator and their significant tests parameters

A sample representation for this analysis is shown in Fig. 5. Each of these charts (a-f) represents one month, (January, April, July, September, October and December, respectively) where these months were selected to show the performance of both Kendal and Sen Methods under various temperature series). All of the charts represents the maximum temperature trend of "6-Qaisumah" Station. Values of $\tau$ and Q were obtained along with their significant parameters and shown on the table included in each chart. The number of stations having significant trends for each month is listed in Table 5, while the number of months having significant trend for each station is shown in Table 6.

\section{DISCUSSION}

As shown in Fig. 5, Kendall and Sen's tests gave similar results with a notice that Sen slope test being more sensitive (rejects more). 
Am. J. Environ. Sci., 6 (2): 191-203, 2010

Table 5: Number of stations with significant increase $T_{\max }, T_{\text {avg }}$ and $\mathrm{T}_{\min }(\mathrm{p}>95 \%)$ for the indicated months as predicted by Sen statistic

\begin{tabular}{lccc}
\hline & \multicolumn{3}{c}{ Number of significant stations } \\
Month & $\mathrm{T}_{\max }$ & $\mathrm{T}_{\text {avg }}$ & $\mathrm{T}_{\min }$ \\
\hline January & 1 & 2 & 1 \\
February & 18 & 10 & 4 \\
March & 20 & 13 & 3 \\
April & 18 & 14 & 8 \\
May & 18 & 14 & 12 \\
June & 19 & 16 & 19 \\
July & 10 & 12 & 20 \\
August & 22 & 21 & 23 \\
September & 18 & 18 & 20 \\
October & 17 & 17 & 13 \\
November & 10 & 3 & 6 \\
December & 4 & 3 & 3 \\
\hline
\end{tabular}

Table 6: Number of months having significant increase in $T_{\max }, T_{\text {avg }}$ and $\mathrm{T}_{\min }(\mathrm{p}>95 \%)$ for each meteorological station according to Sen statistic

\begin{tabular}{|c|c|c|c|c|}
\hline Name & $\mathrm{T}_{\max }$ & $\mathrm{T}_{\text {avg }}$ & $\mathrm{T}_{\min }$ & Index \\
\hline TURAIF & 3 & 1 & 4 & 8 \\
\hline ARAR & 9 & 8 & 5 & 22 \\
\hline GURAIAT* $*$ & 0 & 0 & 0 & 0 \\
\hline AL JOUF & 5 & 3 & 8 & 16 \\
\hline RAFHA & 5 & 3 & 5 & 13 \\
\hline QAISUMAH & 8 & 6 & 6 & 20 \\
\hline TABUK & 1 & 5 & 5 & 11 \\
\hline HAFR AL-BATIN & 1 & 1 & 5 & 7 \\
\hline HAIL & 9 & 9 & 4 & 22 \\
\hline WEJH & 2 & 3 & 4 & 9 \\
\hline GASSIM & 9 & 9 & 2 & 20 \\
\hline DHAHRAN & 8 & 9 & 3 & 20 \\
\hline Dammam* & 0 & 0 & 0 & 0 \\
\hline AHSA & 9 & 2 & 7 & 18 \\
\hline MADINA & 7 & 6 & 6 & 19 \\
\hline RIYADH (New) & 7 & 6 & 6 & 19 \\
\hline RIYADH (Old) & 3 & 8 & 5 & 16 \\
\hline YENBO & 11 & 7 & 4 & 22 \\
\hline JEDDAH & 8 & 5 & 3 & 16 \\
\hline MAKKAH & 0 & 2 & 10 & 12 \\
\hline TAIF & 9 & 2 & 0 & 11 \\
\hline BAHA & 7 & 4 & 2 & 13 \\
\hline W-DAWASIR & 8 & 0 & 6 & 14 \\
\hline BISHA & 11 & 7 & 5 & 23 \\
\hline ABHA & 8 & 10 & 8 & 26 \\
\hline KHAMIS MUSHAIT & 9 & 12 & 9 & 30 \\
\hline NEJRAN & 12 & 7 & 2 & 21 \\
\hline SHARURRAH & 6 & 0 & 1 & 7 \\
\hline GIZAN & 0 & 8 & 7 & 15 \\
\hline
\end{tabular}

A negative $\tau$ or $\mathrm{Q}$ indicates a decreasing trend, January, while a positive sign indicates an increasing trend, April. The significance of $\tau$ was tested by comparing the calculated $\alpha_{\min }$ with $\alpha=0.05$ or 0.01 for 95 and 99\% confidence level, respectively; $\alpha_{\min }<0.05$ or 0.01 . The corresponding significant test for $\mathrm{Q}$ was carried out by calculating its confidence intervals at 95 and $99 \%$ indicated $\left(\mathrm{Q}_{\min }^{95 \%}, \mathrm{Q}_{\max }^{95 \%}\right)$ and $\left(\mathrm{Q}_{\min }^{99 \%}, \mathrm{Q}_{\max }^{99 \%}\right)$, respectively. If the two limits have similar sign, then the calculated Sen Slope Q cannot be zero and therefore the slope is significantly higher than the zero indicating a positive or negative trend.

Figure 5 represents all possible combinations of $\tau$ and $Q$ and their significance. In January, $\tau$ and $Q$ values were negative, indicating a decreasing trend for maximum daily temperature, $\mathrm{T}_{\max }$. But this decrease in not significant according to Kendall $\tau$ since $\alpha_{\min }=0.44$ which is much greater than 0.05 or 0.01 . It is also not significant according to Sen slope Q since the higher limits at $95 \%$ and $99 \%$ were greater than zero $\left(Q_{\text {max }}^{95 \%}=0.087, Q_{\text {max }}^{99 \%}=0.128\right)$. For April both tests showed that $\mathrm{T}_{\max }$ is increasing significantly at $95 \%$ level since $\tau$ and $\mathrm{Q}$ were positive and $\alpha_{\min }=$ $0.0086<0.05$ and $\mathrm{Q}_{\min }^{95 \%}>0.0$. However, while the uptrend is also significant at $99 \%$ level according to Kendall $\tau$, alpha $\min =0.0086<0.01$, it was not significant according to Sen slope at the same level since $99 \% \mathrm{Q}_{\max }^{99 \%}<0.0$ indicating the possibility of the slope being zero. This result showed that the Sen Slope test seemed to be more conservative than Kendall test. Similar results were found for July since the uptrend was significant at $95 \%$ level according to Kendall $\tau$, $\alpha_{\min }=0.032<0.05$ but not significant according to Sen slope , 95\% $\mathrm{Q}_{\min }^{95 \%}=-0.007<0.0$. However, these discrepancies between Kendall and Sen Tests were uncommon and occurred only in few cases. This is shown in the temperature analysis for the flowing three months; September, October and December were both tests gave consistent results. In September, both tests showed that the temperature uptrend is only significant at $95 \%$ level since $\alpha_{\min }=0.0105<0.05$ and $\mathrm{Q}_{\min }^{95 \%}=0.009>0.0$. In October, the uptrend seemed to be significant at both $95 \%$ and $99 \%$ level. In December, the uptrend is not significant at $95 \%$ nor at $99 \%$ as $\alpha_{\min }=0.376>0.05$ and both $\mathrm{Q}_{\min }^{95 \%}$ and $\mathrm{Q}_{\min }^{99 \%}<0.0$.

This analysis was carried out for each month for the studied stations.

Contour maps were plotted to show variation of maximum temperature and its significance for the entire Saudi Arabia for each months (Fig. 6). In Fig. 6, Kendall $\mathrm{Z}$ values as calculated from Equation 6 were used for plotting to show the direction and significance of trends in $T_{\max }$. Positive and significant trends in $\mathrm{T}_{\max }(\mathrm{p}>90 \%)$ were represented by solid grey scale and the level of significance is increasing as gray gets more dark. Negative trends were indicated by dotted surface while white surface indicates no trend. 


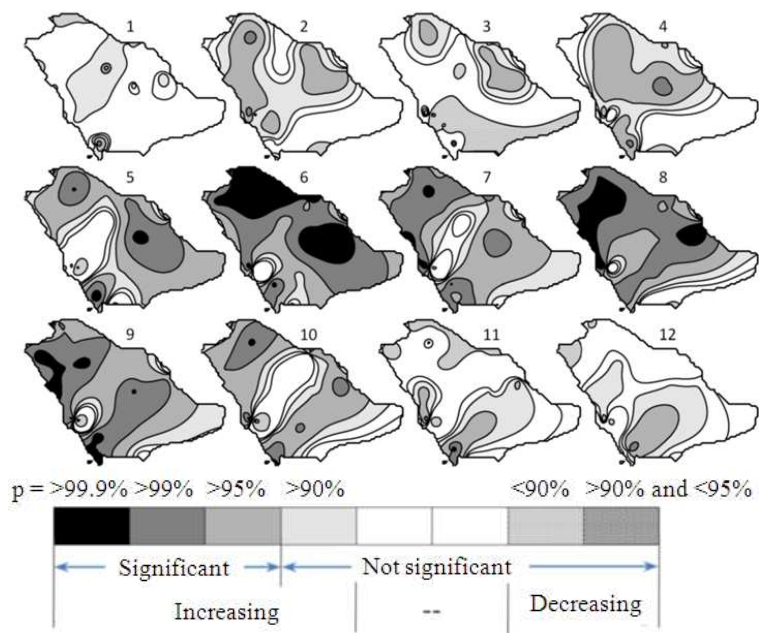

Fig. 6: Contour plots of Kendall $\mathrm{Z}$ statistic for the maximum temperature's trend in various months during the past 29 years

In general a significant warming trends is observed in almost all Saudi Arabia during the whole year except in the winter months of January and November and December to lesser degree. In these three winter months some cooling trends can be noticed, dotted surface, in the northern and eastern part of the country but this cooling is not significant at $90 \%$ probability. Another general observation is that no increase in $\mathrm{T}_{\max }$ was observed in the northern part of the country, white regions, near to the red sea during the year, "7-Tabuk., 10-Wejh." and "6-Qaysoumah. In all other months from February to October, a rising $T_{\max }$ was observed in all parts of Saudi Arabia at varying degree of significance. A high significant warming trend ( $>>99 \%$ ), dark regions, was observed in the month of August, June, May, December, October, April and July in that order and concentrated in the middle, north east and south west belt of the country from "2-Arar., in the North to "27-Najran., in the South. "27-Najran., area in the south seemed to have a warming trend in the whole year including the winter months, November to January. Temperature variations in the Month of July seemed to be different from that in June and August. A significant warming trend was still observed but to a lesser degree $(95 \%<\mathrm{p}<99 \%)$ in most parts of SA except in the North West and North East.

Similar analysis, to that in Fig. 5, was carried out for the entire weather stations in Saudi Arabia for each month and the results are summarized in Table 2-4 for maximum, average and minimum temperatures, respectively. Table 2-4 show the values of Kendall $\tau$ and Sen's Q statistics and an index, white, black or half black circles, indicating the value's significance level. A black circle indicates the value is significant at $99 \%$ level; a half circle is for $95 \%$ significant level, while a white circle indicates no significance at $95 \%$ level.

Results indicated that both Kendall and Sen nonparametric statistics were appropriate to detect trends in temperature time series and produce similar results under 95\% significant level as noticed in Table 2-4. The temperature trends and their significance are fond on Table 2-4 for all studied stations at all months of the year. In general, temperature increase is shown in the summer months from March to October for most of stations. Temperatures in August seemed to rise significantly more than any other months of the year. In the winter months, November, December and January, temperatures are still on the rise for some stations but a decreasing trend was noticed in some others but those positive or negative trends were insignificant. January had the lowest temperatures and showed a decrease in temperatures for about 10 stations and increase in others but those trends were insignificant at $95 \%$ probability level.

Table 5 summarizes the number of stations having warmer trend in each month. The largest temperature increase have been noticed during the month of August where 22, 21 and 23 stations out of 27 showed a significant uptrend in the maximum, average and minimum temperatures, respectively followed by September, October, June and May covering 18, 16 and 17 and 14 stations in average temperature. Similar trends in for $\mathrm{T}_{\max }$ and $\mathrm{T}_{\min }$ were noticed to even a higher degree in some cases. In general, the summer months in Saudi Arabia spanning from May to October seemed to get warmer with time. In winter only 3 stations showed some warming in $\mathrm{T}_{\mathrm{avg}}$ and this warming trend is insignificant.

Table 6 summarizes the number of months with a significant uptrend in temperatures for each station. "13-Dammam. and "3-Guraiat., were neglected from the analysis due to small number of recorded years of data. "27-Nejran., "24-Bisha., 18-Yenbo., "26-Khamis Mushait., 25-Abha., stations showed the highest increase in temperatures in the past 30 years. In "26Khamis-Mushait., an uptrend in the average temperature was recorded for 12 months flowed by the nearby stations of "25-Abha., 10 months and "24Bisha., of 7 months. It is interesting to note that these stations have the highest altitude in the country and considered of mild climate.

Other stations showed similar trends to a lesser degree. About 17 stations out of 27 have warming trends for 7 months or higher. On interesting observation is related to the city of "20-Makkah. Time series analysis revealed that no warming trend was 
observed for the $\mathrm{T}_{\max }$ and only 2 months showed warming trends in the $\mathrm{T}_{\text {avg }}$, but the $\mathrm{T}_{\min }$ have a warming trend for 10 out of 12 months of the year. This is probably due to the special demographic and geographic characteristics of the city, which pushes the minimum temperature higher, but kept the average temperature relatively constant in the past 30 years.

It seemed that the increase in temperature with time is to some extent related to station altitude. A simple regression analyses, shown in Fig. 7, between the number of months having a positive uptrend in the average temperature and station altitude revealed a week, but positive slope suggesting that warming trends in SA is higher in stations having large altitude. Further analysis was carried out to examine the relationship between temperature trends and latitude and the results are shown in Fig. 8. A weak but negative relationship between number of months with up-trends average and maximum temperatures and the corresponding station latitudes was found, Fig. 7. "20-Makkah. and "29Gizan., was not included in the plots because they show no temperature uptrend in any month during the year.

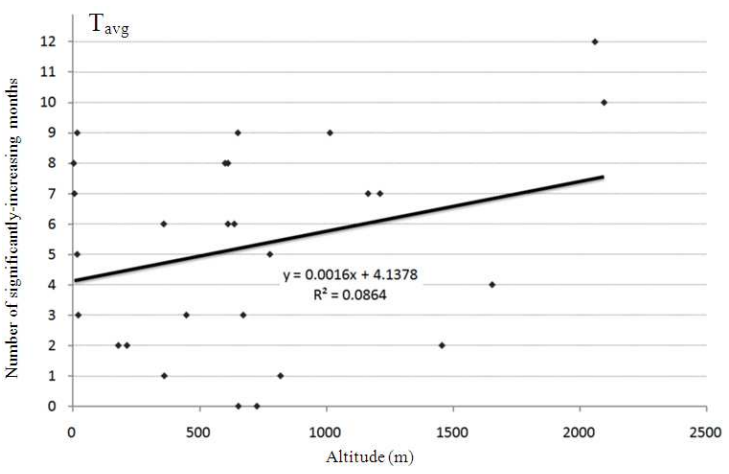

Fig. 7: Relationship between number of months with increasing trend in average temperature and the corresponding stations altitudes

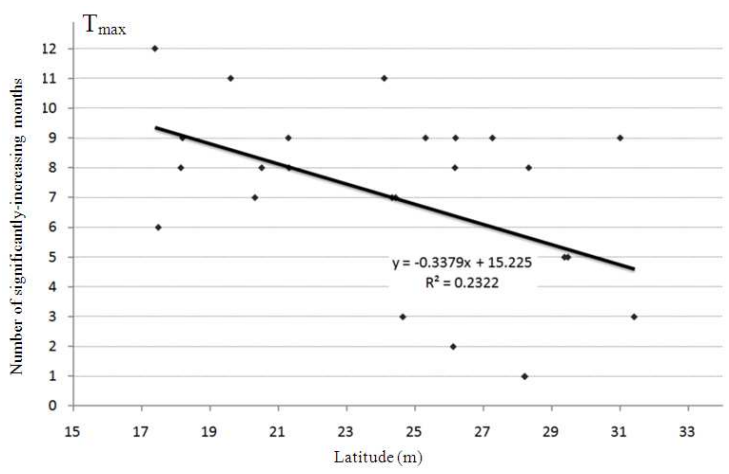

Fig. 8: Relationship between number of months with increasing trend in maximum temperature and the corresponding stations altitudes
Nevertheless, it seemed that temperature is increasing in the southern region of the kingdom at higher rate compared to northern parts as been previously noticed in the contour maps in Fig. 6. Most of the agricultural production and investments are carried out in the southern region of the kingdom, as well as the touristic investments due to the beautiful scenes and mild climate there. Temperature increase in the southern region could have negative impact on tourist activities and agricultural production.

\section{CONCLUSION}

This study investigates temperature changes in KSA for 29 years using data obtained from 29 meteorological stations distributed over the country. The study showed a warming trend in the maximum, minimum and average temperatures throughout the year except in the winter months of November to January where non-significant cooling trends were observed in some stations. Highly significant warming trends were found in June, August and September, in the central region with more than $99 \%$ significance levels. The northwestern and southern regions were the least affected by the warming trend, probably due to the effect of the red sea in the west and the high altitudes in the south. The study confirmed that KSA as well as the Arabian Peninsula were affected by the climate variability, as the temperature tends to rise significantly all over the country in April until October. This raises an important issues related to water resources and agricultural management.

\section{ACKNOWLEDGEMENT}

The authors wish to express their deep thanks and gratitude to "Shaikh Mohammad Bin Husain Alamoudi" for his kind financial support to the King Saud University, http://www.ksu.edu.sa, through the research chair "Shaikh Mohammad Alamoudi Chair for Water Researches" (ACW), http://awc.ksu.edu.sa, where this study is part of the AWC chair activities in the "Projects \& Research" axis. Thanks should also be expressed to the Presidency of Meteorology and Environment in Riyadh, KSA, who kindly provided the meteorological data.

\section{REFERENCES}

Abderrahman, W.A. and I.M. Al-Harazin, 2008. Assessment of climate changes on water resources in the Kingdom of Saudi Arabia. Proceeding of the GCC Environment and Sustainable Development Symposium, Jan. 28-30, Dhahran, Saudi Arabia, Section D-1, pp: 1-13. 
Abramowitz, M. and I.A. Stegun, 1972. Handbook of Mathematical Functions. 10th Edn., Dover Publications Inc., ISBN: 978-0-486-61272-0, pp: 1046.

Albakry, A., I. Alsaleem and M. ElBeishi, 2010. Geography of the Kingdom of Saudi Arabia and Some Other Countries. 3rd Edn., Saudi Ministry of Education, ISBN: 9960-19-056-0, pp: 164.

Al-Zawad, F.M., 2008. Impacts of climate change on water resources in Saudi Arabia. Proceeding of the 3rd International Conference on Water Resources and Arid Environments and the 1st Arab Water Forum, Nov. 16-19, Riyadh, Saudi Arabia, pp: 26. http://www.icwraepsipw.org/images/stories/2008/Environment/10.pdf

Arabia, 2009. In Encyclopedia Britannica. From Encyclopedia Britannica. http://www.britannica.com/EBchecked/topic/31551 /Arabia

Bou-Zeid, E. and M. El-Fadel, 2002. Climate change and water resources in the Middle East: a vulnerability and adaptation assessment. ASCE, J. Water Resour. Plann. Manage., 128: 343-355. DOI: 10.1061/(ASCE)0733-9496(2002)128:5(343)

Can, A. and A.T. Atimtay, 2004. Time series analysis of mean temperature data in Turkey. Proceeding of the 13th World Clean Air and Environmental Protection Congress, Aug. 22-27, London, UK., pp: 6.

Cueto, O.R.G., A.T. Martinez and G.B. Morales, 2009. Urbanization effects upon the air temperature in Mexicali, B.C., Mexico. Atmosfera, 22: 349-365.

Gilbert, R.O., 1987. Statistical methods for environmental pollution monitoring. Van Nostrand Reinhold, New York, ISBN: 978-0-471-28878-7, pp: 336.

Govinda, R.P., 1993. Climatic changes and trends over a major river basin in India. Clim. Res., 2: 215-223.
Isaaks, E.H. and R.M. Srivastava, 1990. An Introduction to Applied Geostatistics. Oxford University Press, Madison Av. NY., ISBN: 0-19505013-v, pp: 560.

Kendall, M., 1975. Multivariate Analysis. Charles Griffin and Company, London.

Kendall, M. and J.D. Gibbons, 1990. Rank Correlation Methods. 5th Edn., Oxford University Press, New York, ISBN: 0-85264-305-5, pp: 272.

Landsberg, H.E., 1975. Sahel drought: Change of climate or part of climate. Theoretical Applied Climatol., 23: 193-200. DOI: 10.1007/BF02246775

Nasrallah, H.A. and R.C. Balling Jr., 1995. Impact of desertification on temperature trends in the Middle East. Environ. Monitor. Assess., 37: 265-271. DOI: 10.1007/BF00546894

Schwartz, P. and D. Randall, 2003. An abrupt climate change scenario and its implications for United States national security. Global Business Network, Emeryville, DOI: 10.1016/j.futures.2004.10.020

Sen, P.K., 1968. Estimates of the regression coefficient based on Kendall's. J. Am. Stat. Assoc., 63: 13791389. DOI: $10.2307 / 2285891$

Smadi, M., 2006. Observed abrupt changes in minimum and maximum temperatures in Jordan in the 20th century. Am. J. Environ. Sci., 2: 114-120.

Smadi, M.M. and A. Zghoul, 2006. A sudden change in rainfall characteristics in Amman, Jordan during the mid 1950s. Am. J. of Environ. Sci., 2: 84-91.

Surfer, 2002. Surfer ${ }^{\circledR}$, a surface mapping system. Windows $^{\mathrm{TM}}$ Software. Golden Software Inc. http://www.goldensoftware.com

$\mathrm{Xu}$, C., L. Gong, T. Jiang, D. Chen and V.P. Singh, 2006. Analysis of spatial distribution and temporal trend of reference evapotranspiration and pan evaporation in Changjiang (Yangtze River) catchment. J. Hydrol., 327: 81-93. DOI: 10.1016/j.jhydrol.2005.11.029 